\title{
Coulomb gauge confinement in the heavy quark limit
}

\author{
C. Popovici, P. Watson, and H. Reinhardt \\ Institut für Theoretische Physik, Universität Tübingen, \\ Auf der Morgenstelle 14, D-72076 Tübingen, Deutschland
}

\begin{abstract}
The relationship between the nonperturbative Green's functions of Yang-Mills theory and the confinement potential is investigated. By rewriting the generating functional of quantum chromodynamics in terms of a heavy quark mass expansion in Coulomb gauge, restricting to leading order in this expansion and considering only the two-point functions of the Yang-Mills sector, the rainbow-ladder approximation to the gap and Bethe-Salpeter equations is shown to be exact in this case and an analytic, nonperturbative solution is presented. It is found that there is a direct connection between the string tension and the temporal gluon propagator. Further, it is shown that for the 4-point quark correlation functions, only confined bound states of color-singlet quark-antiquark (meson) and quark-quark (baryon) pairs exist.
\end{abstract}

PACS numbers: 11.10.St,12.38.Aw

\section{INTRODUCTION}

In quantum chromodynamics $[\mathrm{QCD}]$ there are two primary aspects of the theory that remain to this day elusive: confinement and dynamical chiral symmetry breaking. Both involve the existence of a nontrivial, nonperturbative scale that is generated dynamically. Typically when one works with a physical theory such as QCD, one does not work directly with physical quantities, but rather with (the more readily calculable) gauge-fixed Green's functions. In the process of 'solving' the theory, one must regularize and renormalize at some scale, assigning the various parameters of the theory physical values such that the dynamically generated nonperturbative scale acquires physical meaning. This naturally raises the question: is there a simple relationship between the Green's functions and the nonperturbative physical scale determined externally?

Further to this, Yang-Mills theory as the sub-theory of QCD without quarks does not have any directly observable quantities (the only observables are the glueballs and their spectrum, neither of which can be detected without having a detector made of matter, i.e., quarks and electrons). Yang-Mills theory is known to be confining in the sense that Wilson loops in lattice simulations exhibit an area law at large separations and this area law gives rise to a linearly rising potential, the coefficient of which is the string tension. Now whilst the lattice string tension exists, it has to be related to some physically observable scale in the real world and this has been explicitly done [1]. Unfortunately, the Wilson loop is not particularly amenable to calculation in continuum Yang-Mills theory (for recent work on this subject, see Ref. [2] and references therein), although it is the most natural quantity on the lattice. However, the physical interpretation of the Wilson loop of pure Yang-Mills theory as corresponding to two infinitely heavy quark sources connected via gluon exchange may provide a way of sidestepping the difficulties of considering the (gauge invariant) Wilson loop in the continuum and providing the connection between the (gauge-fixed) Green's functions of Yang-Mills theory and physical quark confinement. The heavy quark mass expansion (and the effective theory associated with it) is a well established area of QCD and indeed uses phenomenologically motivated potentials in place of the Yang-Mills sector [3-5] (see also, for example, Ref. [6]). Moreover, in the heavy quark mass limit the spin of the quark decouples from the system (the so-called heavy quark symmetry) leading to dramatic simplifications.

In full QCD, the Bethe-Salpeter equation is one tool for describing systems composed of quark pairs with interactions given via the Green's functions of the theory. In the light quark sector where one is concerned primarily with dynamical chiral symmetry breaking and the spectrum of the light mesons, covariant gauge Bethe-Salpeter studies have proven to be extremely useful for phenomenology (e.g., Refs. [7, 8] or the contemporary review Ref. [9] and references therein). Such studies are typically restricted to the rainbow-ladder truncation, where the Bethe-Salpeter kernel is reduced to the single exchange of a dressed gluon and so that the problem is tractable. However, it is known that the BetheSalpeter kernel is not so simple and indeed, there has been recently some considerable attention focused on going beyond the rainbow-ladder approximation both in terms of vertex corrections (see for example Refs. [10-15]) and in unquenching effects (e.g., Refs. [11, 16-18]). Whilst more sophisticated kernels do entail a considerable increase in effort, one of the more intriguing results of these studies is that (aside from the meson decay mechanism induced by unquenching [17]) the rainbow-ladder truncation seems surprisingly robust. In Coulomb gauge, there also exist studies of dynamical chiral symmetry breaking and the Bethe-Salpeter equation (see for example Ref. [19] or the more recent Ref. [20] and references therein). Here, potential models (corresponding to the rainbow-ladder truncation) have been used.

As was recognized early on, Coulomb gauge is an ideal choice for studying nonperturbative phenomena [21], but 
because of the inherent noncovariance, significant technical progress has been only recently achieved (mainly in YangMills theory). The Gribov-Zwanziger confinement scenario [22-24] is particularly relevant in Coulomb gauge. In this scenario, the temporal component of the gluon propagator is infrared enhanced (providing a long-range confining force) whereas the transverse spatial components are infrared suppressed (and therefore do not appear as asymptotic states). The relevance of Coulomb gauge stems from the fact that the system reduces naturally to physical degrees of freedom [24]. Using functional methods, it has been formally shown that there exists a conserved and vanishing total color charge (along with the absence of the infamous Coulomb gauge energy divergences) [25]. In addition, the Dyson-Schwinger equations have been explicitly derived [26-28], along with the Slavnov-Taylor identities (for Yang-Mills theory) [29] and one-loop perturbative results have been calculated [27, 28, 30]. On the lattice, there are also initial results for the Yang-Mills propagators now available (although at present the lattice sizes are still modest) [31,32]. Of note is that on the lattice, the temporal gluon propagator appears largely independent of the energy (in the noncovariant Coulomb gauge, the propagators are in general dependent on both the energy and spatial momentum as separate variables, unlike in covariant gauges) and is consistent with a $1 / \vec{q}^{4}$-behavior in the infrared. Furhermore in Coulomb gauge the static spatial lattice gluon propagator is found to vanish in the infrared and to agree with Gribov's formula [31]. The lattice results support the findings of the variational approach to continuum Yang-Mills theory in Coulomb gauge [33, 34] (c.f. also [35, 36]).

To establish a connection between the Green's functions of Yang-Mills theory and the physical world represented by the existence of the string tension that confines quarks, we propose to study here the full QCD Bethe-Salpeter equation with a heavy quark mass expansion at leading order in Coulomb gauge. Given that all quarks are confined irrespective of their mass, it is reasonable to suppose that the existence of the confinement potential and the value of the string tension are independent of the mass and the configuration of the quarks, the only important quantity being their separation in this respect.

Now, the heavy quark mass expansion is clearly not a new concept, nor is the Bethe-Salpeter equation and the existence of a confinement potential. We shall be working in Coulomb gauge and again, there do exist studies of the Bethe-Salpeter equation within this context as discussed above. Thus, we should be specific about what is new in this work. Heavy quark effective theory is done typically in covariant gauges. Separately, in Coulomb gauge the study of the Bethe-Salpeter equation has focused on chiral symmetry breaking and the existence of the confinement potential has been taken as being more or less a settled matter (see for example Ref. [19]). However, the Coulomb gauge Bethe-Salpeter studies have not gone beyond the leading (rainbow-ladder) approximation. In this study, we shall work with heavy quarks in Coulomb gauge and with the emergent results for the explicit Green's functions of the Yang-Mills sector (at least under truncation). In addition we will be working nonperturbatively, deriving and utilizing in part the Slavnov-Taylor identity for the quark-gluon vertex and its peculiar Coulomb gauge form. We thus combine the separate areas of study. What we shall show is that as a result of this combination, the leading (rainbowladder) approximation is exact in the case considered, leading directly to the interpretation of quarks being confined by a linearly rising potential and providing an explicit link between the Green's functions of the nonperturbative Yang-Mills sector and the external physical scale.

The paper is organized as follows. In Section 2 we consider the generating functional of Coulomb gauge QCD at leading order in the heavy quark mass expansion. The tree-level quark and antiquark propagators are derived and the relevant Feynman prescriptions are introduced. In Section 3 the nonperturbative quark gap equation is discussed. Using the Slavnov-Taylor identities in Coulomb gauge (derived explicitly in Appendix A) and truncating the YangMills sector to include only dressed two-point functions, it is found that the rainbow approximation to the quark and antiquark gap equation is nonperturbatively exact. This result is confirmed by the semiperturbative approach considered in Section 4. In Sections $5 \& 6$, the Bethe-Salpeter equations for quark-antiquark and diquark states are considered. It is shown that the ladder approximation to the Bethe-Salpeter kernel is nonperturbatively exact and that only color-singlet meson and $S U(2)$ baryon states have finite energy solutions. Moreover, a direct connection between the temporal gluon propagator and the string tension is established, at least under the truncation scheme considered.

\section{HEAVY QUARK MASS EXPANSION}

In this study, we are concerned with combining nonperturbative physics with the heavy quark mass expansion. The two are not automatically compatible; for example, whilst the mass expansion assumes that the mass parameter is the largest scale in the problem, loop integrals involve momenta up to the UV-cutoff scale (supposing for the moment that a UV-cutoff regularization is employed) which is also assumed to the the largest scale. In heavy quark effective theory [HQET], the recognition of this apparent contradiction leads to the so-called matching procedure [3]. This is just one example of the effect of the heavy mass expansion and some care must be taken in proceeding. The overriding concern here is that we wish to use wherever possible the full nonperturbative QCD functional formalism, i.e., the 
gap and Bethe-Salpeter equations and Slavnov-Taylor identities for the complete quark fields, rather than HQET expressions that refer to the heavy quark degrees of freedom. The effect will be (see below) that we are obliged to restrict ourselves to leading order in the mass expansion. This naturally prohibits a quantitative analysis involving real quarks (which are not infinitely heavy) but does not deflect from the goal of studying the relationship between the string tension and the Yang-Mills Green's functions.

Throughout this work, we shall use the conventions and notations established previously in Refs. [26-29]. We work in Minkowski space with the metric $g_{\mu \nu}=\operatorname{diag}(1,-\overrightarrow{1})$ and all minus signs associated with the spatial components of covariant and contravariant vectors are explicitly extracted. Roman sub/superscripts $(i, j, \ldots)$ denote spatial indices, superscripts $(a, b, \ldots)$ denote color indices in the adjoint representation. Configuration space coordinates may be denoted with subscript $(x, y, \ldots)$ when no confusion arises. Other index notation will be explained as the context arises. The Dirac $\gamma$-matrices satisfy $\left\{\gamma^{\mu}, \gamma^{\nu}\right\}=2 g^{\mu \nu}$. The explicit quark contribution to the full QCD generating functional is $[28]$

$$
\begin{aligned}
Z[\bar{\chi}, \chi]= & \int \mathcal{D} \Phi \exp \left\{\imath \int d^{4} x \bar{q}_{\alpha}(x)\left[\imath \gamma^{0} D_{0}+\imath \vec{\gamma} \cdot \vec{D}-m\right]_{\alpha \beta} q_{\beta}(x)\right\} \\
& \times \exp \left\{\imath \int d^{4} x\left[\bar{\chi}_{\alpha}(x) q_{\alpha}(x)+\bar{q}_{\alpha}(x) \chi_{\alpha}(x)\right]+\imath \mathcal{S}_{Y M}\right\}
\end{aligned}
$$

with the temporal and spatial components of the covariant derivative (in the fundamental color representation) given by

$$
\begin{aligned}
D_{0} & =\partial_{0}-\imath g T^{a} \sigma^{a}(x), \\
\vec{D} & =\vec{\nabla}+\imath g T^{a} \vec{A}^{a}(x) .
\end{aligned}
$$

In the above, $\mathcal{D} \Phi$ generically denotes the functional integration measure over all fields present. $q_{\alpha}(x)$ denotes the full quark field ( $\bar{q}$ is the conjugate or antiquark field) where in this case, the subscript $\alpha$ refers to the fundamental color, flavor and spin attributes collectively. The sources $\chi_{\alpha}(x)$ and $\bar{\chi}_{\alpha}(x)$ are for the complete quark fields. The Yang-Mills contribution to the generating functional (which will only be of direct relevance to this study in Appendix A) is in the standard, second order formalism [27, 29]. Additionally, $\vec{A}$ and $\sigma$ refer to the spatial and temporal components of the gluon field, respectively. The $T^{a}$ are the (Hermitian) generators of the $S U\left(N_{c}\right)$ group satisfying $\left[T^{a}, T^{b}\right]=\imath f^{a b c} T^{c}$ (with the fully antisymmetric structure constants $f^{a b c}$ ) and normalized via $\operatorname{Tr}\left(T^{a} T^{b}\right)=\delta^{a b} / 2$. For later use we introduce the color factor associated with the quark self-energy:

$$
C_{F}=\frac{N_{c}^{2}-1}{2 N_{c}} .
$$

Now consider the following decomposition of the quark and antiquark fields:

$$
\begin{array}{ccc}
q_{\alpha}(x)=e^{-\imath m x_{0}}[h(x)+H(x)]_{\alpha}, & h_{\alpha}(x)=e^{\imath m x_{0}}\left[P_{+} q(x)\right]_{\alpha}, \quad H_{\alpha}(x)=e^{\imath m x_{0}}\left[P_{-} q(x)\right]_{\alpha} \\
\bar{q}_{\alpha}(x)=e^{\imath m x_{0}}[\bar{h}(x)+\bar{H}(x)]_{\alpha}, \quad \bar{h}_{\alpha}(x)=e^{-\imath m x_{0}}\left[\bar{q}(x) P_{+}\right]_{\alpha}, \bar{H}_{\alpha}(x)=e^{-\imath m x_{0}}\left[\bar{q}(x) P_{-}\right]_{\alpha}
\end{array}
$$

where the (spinor) projection operators are

$$
P_{ \pm}=\frac{1}{2}\left(\mathbb{1} \pm \gamma^{0}\right), \quad P_{+}+P_{-}=\mathbb{1}, \quad P_{+} P_{-}=0, \quad P_{ \pm}^{2}=P_{ \pm} .
$$

This decomposition is a particular case of the heavy quark transform underlying HQET [3]. There, one recognizes that a heavy quark within a hadron is almost on-shell and moves with the hadron velocity $v$ such that the 4-momentum can be written $p^{\mu}=m v^{\mu}+k^{\mu}$ where $|k| \ll m|v|$ and $v^{2}=1$ (such that when $|k|=0, p^{2}=m^{2}$ ). One then uses the general projectors $P_{ \pm}=(\mathbb{1} \pm \psi) / 2$ and with the exponential terms $e^{ \pm \imath m v \cdot x}$. The case used here corresponds to the rest frame of the quark, $v^{\mu}=(1, \overrightarrow{0})$, but within the context of the generating functional is simply a choice of (arbitrary) decomposition that will prove useful in Coulomb gauge. In fact, this choice will result in the simplification whereby the spatial components of the Yang-Mills Green's functions are absent at leading order in the mass expansion. The virtue of the heavy quark decomposition is that the projection operators satisfy the following further relations

$$
P_{+} \gamma^{0} P_{+}=P_{+} P_{+}, \quad P_{+} \gamma^{0} P_{-}=0, \quad P_{+} \gamma^{i} P_{+}=0
$$

such that the following relations hold for the components of the quark field:

$$
\bar{h} \gamma^{0} h=\bar{h} h, \bar{H} \gamma^{0} H=-\bar{H} H, \quad \bar{h} \gamma^{0} H=\bar{H} \gamma^{0} h=\bar{h} \gamma^{i} h=\bar{H} \gamma^{i} H=0 .
$$


Inserting the decomposition of the quark fields given by Eq. (2.4) into the generating functional Eq. (2.1) and using these relationships one obtains

$$
\begin{aligned}
Z[\bar{\chi}, \chi]= & \int \mathcal{D} \Phi \exp \left\{\imath \int d ^ { 4 } x \left[\bar{h}_{\alpha}(x)\left[\imath D_{0}\right]_{\alpha \beta} h_{\beta}(x)\right.\right. \\
& \left.\left.+\bar{h}_{\alpha}(x)[\imath \vec{\gamma} \cdot \vec{D}]_{\alpha \beta} H_{\beta}(x)+\bar{H}_{\alpha}(x)[\imath \vec{\gamma} \cdot \vec{D}]_{\alpha \beta} h_{\beta}(x)+\bar{H}_{\alpha}(x)\left[-2 m-\imath D_{0}\right]_{\alpha \beta} H_{\beta}(x)\right]\right\} \\
& \times \exp \left\{\imath \int d^{4} x\left[e^{-\imath m x_{0}} \bar{\chi}_{\alpha}(x)[h(x)+H(x)]_{\alpha}+e^{\imath m x_{0}}[\bar{h}(x)+\bar{H}(x)]_{\alpha} \chi_{\alpha}(x)\right]+\imath \mathcal{S}_{Y M}\right\} .
\end{aligned}
$$

The difference between the mass expansion (as used here) and HQET can now be explained. Because of our insistence on retaining the source terms for the full quark fields, our generating functional has only been rewritten in terms of different integration variables (note that the Jacobian of the transform is field independent and thus trivial) and not altered, although the source term expression in the above is slightly modified by the appearance of the exponential factors. We will thus be able to work with the full gap and Bethe-Salpeter equations for quarks which are derived from $Z[\bar{\chi}, \chi]$. This is in contrast to HQET where the quark sources are replaced with sources for the $h$-fields (the $H$-fields are integrated out as below). The differences are mainly cosmetic at the level of this study (which will concentrate on the leading order in the mass expansion, see below) but serve to illustrate some useful points which will be highlighted as they arise.

The point of the heavy quark decomposition of the fields is that for the $h$-field ('large') components, the quark mass parameter $m$ does not appear directly. When we integrate out the $H$-fields however, we get the following expression

$$
\begin{aligned}
Z[\bar{\chi}, \chi]= & \int \mathcal{D} \Phi \operatorname{Det}\left[\imath D_{0}+2 m\right] \exp \left\{\imath \int d ^ { 4 } x \left[\bar{h}_{\alpha}(x)\left[\imath D_{0}\right]_{\alpha \beta} h_{\beta}(x)\right.\right. \\
& \left.\left.+\left[\bar{h}(x) \imath \vec{\gamma} \cdot \vec{D}+e^{-\imath m x_{0}} \bar{\chi}(x)\right]_{\alpha}\left[\imath D_{0}+2 m\right]_{\alpha \beta}^{-1}\left[\imath \vec{\gamma} \cdot \vec{D} h(x)+e^{\imath m x_{0}} \chi(x)\right]_{\beta}\right]\right\} \\
& \times \exp \left\{\imath \int d^{4} x\left[e^{-\imath m x_{0}} \bar{\chi}_{\alpha}(x) h_{\alpha}(x)+e^{\imath m x_{0}} \bar{h}_{\alpha}(x) \chi_{\alpha}(x)\right]+\imath \mathcal{S}_{Y M}\right\} .
\end{aligned}
$$

Obviously, since we have integrated out a nontrivial component of the original quark field, our expression is nonlocal and this is where the heavy mass expansion is necessary. We notice that [3]

$$
\begin{aligned}
\operatorname{Det}\left[\imath D_{0}+2 m\right] & \sim \mathcal{O}\left(1 / m^{2}\right) \\
{\left[\imath D_{0}+2 m\right]_{\alpha \beta}^{-1} X_{\beta} } & \sim \frac{1}{2 m} X_{\alpha}+\mathcal{O}\left(1 / m^{2}\right) .
\end{aligned}
$$

Leaving the $e^{ \pm \imath m x_{0}}$ factors as they are, we can thus write

$$
\begin{aligned}
Z[\bar{\chi}, \chi]= & \int \mathcal{D} \Phi \exp \left\{\imath \int d^{4} x\left[\bar{h}_{\alpha}(x)\left[\imath D_{0}\right]_{\alpha \beta} h_{\beta}(x)+\frac{1}{2 m}\left[\bar{h}(x) \imath \vec{\gamma} \cdot \vec{D}+e^{-\imath m x_{0}} \bar{\chi}(x)\right]_{\alpha}\left[\imath \vec{\gamma} \cdot \vec{D} h(x)+e^{\imath m x_{0}} \chi(x)\right]_{\alpha}\right]\right\} \\
& \times \exp \left\{\imath \int d^{4} x\left[e^{-\imath m x_{0}} \bar{\chi}_{\alpha}(x) h_{\alpha}(x)+e^{\imath m x_{0}} \bar{h}_{\alpha}(x) \chi_{\alpha}(x)\right]+\imath \mathcal{S}_{Y M}\right\}+\mathcal{O}\left(1 / m^{2}\right) .
\end{aligned}
$$

Our generating functional is now local in the fields and arranged in an expansion in the parameter $1 / m$ (this will be referred to as the mass expansion although strictly speaking, it is an expansion in the inverse mass). However, locality does not mean that the above expression can be directly applied. Let us consider the classical (full) quark field in the presence of sources:

$$
\begin{aligned}
& \frac{1}{Z} \int \mathcal{D} \Phi q_{\alpha}(x) \exp \{\imath \mathcal{S}\}=\frac{1}{Z} \frac{\delta Z}{\delta \imath \bar{\chi}_{\alpha}(x)} \\
& \quad=\frac{1}{Z} \int \mathcal{D} \Phi\left\{e^{-\imath m x_{0}} h_{\alpha}(x)+\frac{e^{-\imath m x_{0}}}{2 m}\left[\imath \vec{\gamma} \cdot \vec{D} h(x)+e^{\imath m x_{0}} \chi(x)\right]_{\alpha}\right\} \exp \{\imath \mathcal{S}\}+\mathcal{O}\left(1 / m^{2}\right) .
\end{aligned}
$$

One sees immediately that even at $\mathcal{O}(1 / m)$, the classical quark field has components that involve interaction type terms $(\vec{D} h \sim \vec{A} h)$ brought about by the truncation of the nonlocality. Of course, this is nothing more than the statement that the $h$-field is nontrivially (and dynamically) related to the full $q$-field. It also means that if we want to use the nonperturbative gap and Bethe-Salpeter equations (i.e., those equations derived from the action for the 
full quark fields $q$ and their sources $\chi$ and which are the equations of QCD as opposed to HQET) then we cannot expect that the mass expansion can realistically be extended far beyond the leading order in order to do practical calculations. As stated previously though, the aim is to investigate the connection between the Yang-Mills sector and the physical world made of quarks; the string tension that represents our goal is not dependent on the quark mass (both light and heavy quarks are confined in the same way, as far as we know). Therefore, we restrict our attention to the leading order in the mass expansion as follows (and writing $D_{0}$ explicitly):

$$
\begin{aligned}
Z[\bar{\chi}, \chi]= & \int \mathcal{D} \Phi \exp \left\{\imath \int d^{4} x \bar{h}_{\alpha}(x)\left[\imath \partial_{0 x}+g T^{a} \sigma^{a}(x)\right]_{\alpha \beta} h_{\beta}(x)\right\} \\
& \times \exp \left\{\imath \int d^{4} x\left[e^{-\imath m x_{0}} \bar{\chi}_{\alpha}(x) h_{\alpha}(x)+e^{\imath m x_{0}} \bar{h}_{\alpha}(x) \chi_{\alpha}(x)\right]+\imath \mathcal{S}_{Y M}\right\}+\mathcal{O}(1 / m) .
\end{aligned}
$$

The standard machinery of functional methods is now employed. From the observation that up to boundary terms (which are assumed to vanish) the integral of a derivative vanishes, we have the quark field equation of motion:

$$
\begin{aligned}
0 & =\int \mathcal{D} \Phi \frac{\delta}{\delta \imath \bar{h}_{\alpha}(x)} \exp \{\imath \mathcal{S}\} \\
& =\int \mathcal{D} \Phi\left\{\left[\imath \partial_{0 x}+g T^{a} \sigma^{a}(x)\right]_{\alpha \beta} h_{\beta}(x)+e^{\imath m x_{0}} \chi_{\alpha}(x)\right\} \exp \{\imath \mathcal{S}\}+\mathcal{O}(1 / m)
\end{aligned}
$$

The field equation of motion for the antiquark gives equivalent results and can be neglected. The generating functional of connected Green's functions (for the full quarks) is defined via $Z=e^{W}$. Denoting the derivatives with respect to sources of $W[\bar{\chi}, \chi]$ with an obvious bracket notation, the classical fields are:

$$
\begin{aligned}
& q_{\alpha}(x)=\frac{1}{Z} \int \mathcal{D} \Phi q_{\alpha}(x) \exp \{\imath \mathcal{S}\}=\frac{1}{Z} \frac{\delta Z[\bar{\chi}, \chi]}{\delta \imath \bar{\chi}_{\alpha}(x)}=:<\imath \bar{\chi}_{\alpha}(x)> \\
& \bar{q}_{\alpha}(x)=\frac{1}{Z} \int \mathcal{D} \Phi \bar{q}_{\alpha}(x) \exp \{\imath \mathcal{S}\}=-\frac{1}{Z} \frac{\delta Z[\bar{\chi}, \chi]}{\delta \imath \chi_{\alpha}(x)}=:-<\imath \chi_{\alpha}(x)>.
\end{aligned}
$$

Also ( $\rho$ is the source for the $\sigma$-field, implicit within $Z$ and $W$ ),

$$
\int \mathcal{D} \Phi \sigma^{a}(x) q_{\alpha}(x) \exp \{\imath \mathcal{S}\}=\frac{\delta^{2} Z[\bar{\chi}, \chi]}{\delta \imath \rho^{a}(x) \delta \imath \bar{\chi}_{\alpha}(x)}=Z[\bar{\chi}, \chi]\left[<\imath \rho^{a}(x) \imath \bar{\chi}_{\alpha}(x)>+<\imath \rho^{a}(x)><\imath \bar{\chi}_{\alpha}(x)>\right]
$$

Notice that from Eq. (2.13), the following relations hold:

$$
\frac{\delta Z[\bar{\chi}, \chi]}{\delta \imath \bar{\chi}_{\alpha}(x)}=\int \mathcal{D} \Phi e^{-\imath m x_{0}} h_{\alpha}(x) \exp \{\imath \mathcal{S}\}+\mathcal{O}(1 / m), \quad \frac{\delta Z[\bar{\chi}, \chi]}{\delta \imath \chi_{\alpha}(x)}=-\int \mathcal{D} \Phi e^{\imath m x_{0}} \bar{h}_{\alpha}(x) \exp \{\imath \mathcal{S}\}+\mathcal{O}(1 / m)
$$

(recalling the earlier discussion of neglecting the $\mathcal{O}(1 / m)$ terms) such that the field equation of motion, Eq. (2.14), can be written in terms of derivatives of $W$ :

$$
\begin{aligned}
0= & {\left[\imath \partial_{0 x}\right]_{\alpha \beta} e^{\imath m x_{0}}<\imath \bar{\chi}_{\beta}(x)>+\left[g T^{a}\right]_{\alpha \beta} e^{\imath m x_{0}}\left[<\imath \rho^{a}(x) \imath \bar{\chi}_{\beta}(x)>+<\imath \rho^{a}(x)><\imath \bar{\chi}_{\beta}(x)>\right]+e^{\imath m x_{0}} \chi_{\alpha}(x) } \\
& +\mathcal{O}(1 / m) .
\end{aligned}
$$

Factoring out the exponential terms gives then

$$
0=\left[\imath \partial_{0 x}-m\right]_{\alpha \beta}<\imath \bar{\chi}_{\beta}(x)>+\left[g T^{a}\right]_{\alpha \beta}\left[<\imath \rho^{a}(x) \imath \bar{\chi}_{\beta}(x)>+<\imath \rho^{a}(x)><\imath \bar{\chi}_{\beta}(x)>\right]+\chi_{\alpha}(x)+\mathcal{O}(1 / m) .
$$

To continue, we make a Legendre transform in order to construct the effective action for the full quark fields. Explicitly separating the quark and Yang-Mills components:

$$
\Gamma[\Phi, \bar{q}, q]=W[J, \bar{\chi}, \chi]-\imath J_{\alpha} \Phi_{\alpha}-\imath \bar{\chi}_{\alpha} q_{\alpha}-\imath \bar{q}_{\alpha} \chi_{\alpha}
$$

such that

$$
\begin{aligned}
q_{\alpha}(x)=<\imath \bar{\chi}_{\alpha}(x)>, & \chi_{\alpha}(x)=-<\imath \bar{q}_{\alpha}(x)>, \\
\bar{q}_{\alpha}(x)=-<\imath \chi_{\alpha}(x)>, & \bar{\chi}_{\alpha}(x)=<\imath q_{\alpha}(x)>, \\
\sigma^{a}(x)=<\imath \rho^{a}(x)>, & \rho^{a}(x)=-<\imath \sigma^{a}(x)>
\end{aligned}
$$


where the same bracket notation for derivatives of the effective action with respect to the classical fields is used (there is no confusion between the two sets of brackets since the two sets of derivatives are never mixed). $J_{\alpha}$ and $\Phi_{\alpha}$ denote generic gluonic (Yang-Mills) sources and classical fields, respectively; the index $\alpha$ here refers to all attributes of the (gluonic) object in question (including its type and position argument) and we use the common convention that this index is either summed or integrated over as appropriate. The field equation of motion can then be rewritten in terms of derivatives of the effective action (which are the proper Green's functions when sources are set to zero):

$$
<\imath \bar{q}_{\alpha}(x)>=\left[\imath \partial_{0 x}-m\right]_{\alpha \beta} q_{\beta}(x)+\left[g T^{a}\right]_{\alpha \beta}\left[<\imath \rho^{a}(x) \imath \bar{\chi}_{\beta}(x)>+\sigma^{a}(x) q_{\beta}(x)\right]+\mathcal{O}(1 / m) .
$$

With the field equation of motion written in the above forms, we can now derive the Feynman rules for the quark components of the theory (the Yang-Mills parts are already known [27]). We start with the quark propagator. From Eq. (2.19), ignoring interaction terms and functionally differentiating we get the tree-level propagator in configuration space

$$
0=\left[\imath \partial_{0 x}-m\right]_{\alpha \beta}<\imath \chi_{\gamma}(z) \imath \bar{\chi}_{\beta}(x)>^{(0)}-\imath \delta_{\gamma \alpha} \delta(z-x)+\mathcal{O}(1 / m) .
$$

We now write the Fourier transforms to define both the anti- and quark propagators, respectively, utilizing the translational invariance:

$$
\begin{aligned}
& <\imath \chi_{\gamma}(z) \imath \bar{\chi}_{\beta}(x)>\equiv W_{q \bar{q} \gamma \beta}(z-x)=\int d k e^{-\imath k \cdot(z-x)} W_{q \bar{q} \gamma \beta}(k), \\
& <\imath \bar{\chi}_{\beta}(z) \imath \chi_{\alpha}(x)>\equiv W_{\bar{q} q \beta \alpha}(z-x)=\int d k e^{-\imath k \cdot(z-x)} W_{\bar{q} q \beta \alpha}(k)
\end{aligned}
$$

such that in momentum space, we get

$$
\begin{aligned}
& 0=\int d k e^{-\imath k \cdot(z-x)}\left\{\left[-k_{0}-m\right]_{\alpha \beta} W_{q \bar{q} \gamma \beta}^{(0)}(k)-\imath \delta_{\gamma \alpha}\right\}+\mathcal{O}(1 / m), \\
& 0=\int d k e^{-\imath k \cdot(z-y)}\left\{W_{\bar{q} q \beta \alpha}^{(0)}(k)\left[k_{0}-m\right]_{\alpha \gamma}+\imath \delta_{\beta \gamma}\right\}+\mathcal{O}(1 / m) .
\end{aligned}
$$

Naively, one would write the solutions as

$$
W_{\bar{q} q \beta \alpha}^{(0)}(k)=\frac{-\imath \delta_{\beta \alpha}}{\left[k_{0}-m\right]}+\mathcal{O}(1 / m), \quad W_{q \bar{q} \gamma \beta}^{(0)}(k)=\frac{-\imath \delta_{\gamma \beta}}{\left[k_{0}+m\right]}+\mathcal{O}(1 / m) .
$$

However, in order to define the Fourier transform, one must first define a prescription for handling the poles in the energy integral. Unlike the conventional tree-level quark propagator, we do not have a pair of simple poles in the complex $k_{0}$-plane, we have instead single poles and this is due to the mass expansion. For the quark propagator, we write

$$
W_{\bar{q} q \beta \alpha}^{(0)}(k)=\frac{-\imath \delta_{\beta \alpha}}{\left[k_{0}-m+\imath \varepsilon\right]}+\mathcal{O}(1 / m) .
$$

Notice the following. The tree-level quark propagator is a scalar quantity (or rather, diagonal in the outer product of the fundamental color, flavor and spinor spaces) as a consequence of the mass expansion. This is one of the most striking features of HQET and physically corresponds to the decoupling of the spin from the heavy quark system. $W_{\bar{q} q}^{(0)}$ is identical to the heavy quark tree-level propagator [3] up to the appearance of the mass term. In HQET, where one uses sources for the $h$-fields directly, this term does not appear and is simply due to a shift of the energy by an amount $m$ (such that one is working with the 'small' momenta around the rest mass energy). We retain this term for completeness in order to see what effect it may have - the Yang-Mills sector may not be compatible with such a shift in the energy, although this will turn out not to be the case. Also, note that the kinetic term of the tree-level propagator would read $-\vec{k}^{2} / 2 m$ in the denominator factor and is at higher order in the mass expansion. Such terms are obviously important to the UV properties of the loop integrals but will not play any role here since we shall be interested in the infrared limit.

Returning to the issue of the Feynman prescription, one of the direct consequences of the single energy pole is that when one considers a closed quark loop (a virtual quark-antiquark pair) at lowest order, the energy integral automatically vanishes (see also Ref. [3] for an alternate discussion on this topic), i.e.,

$$
\int \frac{d k_{0}}{\left[k_{0}-m+\imath \varepsilon\right]\left[k_{0}+p_{0}-m+\imath \varepsilon\right]}=0 .
$$


It can be justified that all such closed quark loops vanish in similar fashion (indeed, precisely this type of integral will be heavily used in the following Sections); in other words, the heavy mass expansion at leading order is quenched. One interpretation of the above result is that due to the presence of only the single pole, the heavy quark can only propagate forward in time and the corresponding antiquark is not present. This leads to a conundrum: how can we construct a quark-antiquark pair in the Bethe-Salpeter equation? The resolution is straightforward - in the Bethe-Salpeter equation one is not considering a virtual quark-antiquark pair but rather a system composed of two separate unphysical particles. It is known that in Coulomb gauge, Gauss' law forbids the creation of a colored state in isolation (the total color charge is conserved and vanishing [25]) and so, the Feynman prescription for the quark (or the antiquark) propagator has no physical meaning in isolation. The quenching of the theory means that closed quark loops vanish and from covariant gauge Bethe-Salpeter studies, the connection between such loops and the Bethe-Salpeter kernel is qualitatively understood [17]. In the quenched case, the quark and antiquark lines of the Bethe-Salpeter equation are never connected by a primitive vertex (unlike the closed quark loop). Another way of seeing this is to consider the flavor nonsinglet Bethe-Salpeter equation - in other words the quark and antiquark constituents considered as two distinct flavors. Because the quark and antiquark are genuinely separate (unphysical) particles, we are at liberty to choose that the antiquark propagator in the Bethe-Salpeter equation has the opposite Feynman prescription as follows:

$$
W_{q \bar{q} \gamma \beta}^{(0)}(k)=\frac{-\imath \delta_{\gamma \beta}}{\left[k_{0}+m+\imath \varepsilon\right]}+\mathcal{O}(1 / m)
$$

As shall be seen, this results in a physical interpretation for the quark-antiquark Bethe-Salpeter equation as a whole. Another way of viewing the above is to realise that the four components of the full quark Dirac equation (quark and antiquark moving either forwards or backwards in time according to causality) have been separated by the mass expansion (the 'large' $h$ or 'small' $H$ field components) and the breaking of the time reversal symmetry. In the context of the mass expansion, there is only the quark or antiquark moving forward in time corresponding to the above Feynman prescriptions. The closed quark loop involves a quark going backwards in time (similarly for an antiquark loop) which is prohibited, or more precisely suppressed by the mass, so that such closed quark loop integrals vanish at leading order whereas the quark-antiquark Bethe-Salpeter equation has a physical solution.

For the proper two-point and three-point functions, we use functional derivatives of Eq. (2.22) and we get directly in momentum space

$$
\begin{array}{cl}
\Gamma_{\bar{q} q \alpha \beta}^{(0)}(k)=\imath\left[k_{0}-m\right] \delta_{\alpha \beta}+\mathcal{O}(1 / m), & \Gamma_{q \bar{q} \alpha \beta}^{(0)}(k)=\imath\left[k_{0}+m\right] \delta_{\alpha \beta}+\mathcal{O}(1 / m), \\
\Gamma_{\bar{q} q \sigma \alpha \beta}^{(0) a}\left(k_{1}, k_{2}, k_{3}\right)=\left[g T^{a}\right]_{\alpha \beta}+\mathcal{O}(1 / m), & \Gamma_{q \bar{q} \sigma \alpha \beta}^{(0) a}\left(k_{1}, k_{2}, k_{3}\right)=-\left[g T^{a}\right]_{\beta \alpha}+\mathcal{O}(1 / m) .
\end{array}
$$

Importantly, the tree-level spatial quark-gluon vertex does not appear at leading order in the mass expansion:

$$
\Gamma_{\bar{q} q A \alpha \beta i}^{(0) a}=\Gamma_{q \bar{q} A \alpha \beta i}^{(0) a}=\mathcal{O}(1 / m) .
$$

Notice the ordering of the indices for the $\Gamma_{q \bar{q} \sigma}$ vertex. Also notice that $\Gamma_{\bar{q} q \alpha \beta}^{(0)}(k)=-\Gamma_{q \bar{q} \alpha \beta}^{(0)}(-k)$ since the two-point function requires no Feynman prescription and is diagonal in the outer product of the fundamental color, flavor and spinor spaces. In addition, $W_{\bar{q} q} \Gamma_{\bar{q} q}=\mathbb{1}$ as usual.

Now, because of our insistence of using the full quark sources, all the nonperturbative equations involving the quarks (the Dyson-Schwinger equations for two-point and three-point functions, the Slavnov-Taylor identities and the Bethe-Salpeter equation) will not alter their form at leading order in the mass expansion (beyond leading order, as previously illustrated, the nontrivial classical field and the resolution of the Legendre transform would play a role). The only alterations are that the tree-level factors have been changed and we demand that only the leading order terms in the mass expansion of the resulting equations are retained. Obviously, in order to solve the nonperturbative system we must further specify our truncation scheme. Recall that at leading order in the mass expansion, the theory is already quenched. The truncation scheme we propose is to consider only the dressed two-point functions of the Yang-Mills sector (i.e., the nonperturbative gluon propagators derived from a hypothetical solution of the complete Yang-Mills theory). This amounts to setting all the pure Yang-Mills vertices and higher $n$-point functions occurring in the quark equations to zero. It is worth pointing out that because of the mass expansion, any loop diagram with a tree-level spatial quark-gluon vertex will be suppressed, such that the number of loop diagrams arising because of the Yang-Mills vertices is heavily restricted and those loops that do contribute will not include the leading order perturbative corrections (the fully temporal gluon Green's functions $\Gamma_{\sigma \sigma \sigma}, \Gamma_{\sigma \sigma \sigma \sigma}, \ldots$ are zero at tree-level). The most important physical point that will emerge is that when we set the Yang-Mills vertices to zero, we exclude the nonAbelian part of the charge screening mechanism of the quark color charge and any potential glueball states. On the other hand, the charge screening mechanism and glueball contributions of the gluon field (i.e., the color string) is implicitly encoded in the nonperturbative form of the temporal gluon propagator. 
With the truncation scheme as outlined above, the Yang-Mills sector collapses to the inclusion of a single object: the temporal gluon propagator which is written as [27]

$$
W_{\sigma \sigma}^{a b}(k)=\delta^{a b} \frac{\imath}{\vec{k}^{2}} D_{\sigma \sigma}\left(\vec{k}^{2}\right) .
$$

There are three important features to this propagator. Firstly, there are indications on the lattice that the dressing function $D_{\sigma \sigma}$ is largely independent of energy [32], justifying the energy independence of the above form. Indeed on general grounds, the temporal gluon propagator must have some part that is constant in the energy in order to cancel closed ghost loops and resolve the Coulomb gauge energy divergences (see also the more formal considerations of Ref. [37]). Second, the lattice analysis indicates that the dressing function $D_{\sigma \sigma}$ is infrared divergent and is likely to behave as $1 / \vec{k}^{2}$ for vanishing $\vec{k}^{2}$. We are interested mainly in the relationship between $D_{\sigma \sigma}$ (as the input of the Yang-Mills sector) and the string tension so we will not need the specific form until towards the end. Third, the product $g^{2} D_{\sigma \sigma}$ is a renormalization group invariant quantity and thus a natural candidate for being relevant to the physical string tension $[24,29]$.

\section{GAP EQUATION: NONPERTURBATIVE TREATMENT}

Let us begin by considering the Dyson-Schwinger equation for the quark two-point proper function (the gap equation). In full QCD (i.e., second order formalism, Coulomb gauge without the mass expansion and derived from the first order formalism results of Ref. [28]), it reads $\left(d \omega=d^{4} \omega /(2 \pi)^{4}\right)$ :

$$
\begin{aligned}
\Gamma_{\bar{q} q \alpha \delta}(k)=\Gamma_{\bar{q} q \alpha \delta}^{(0)}(k)+\int d \omega & \left\{\Gamma_{\bar{q} q \sigma \alpha \beta}^{(0) a}(k,-\omega, \omega-k) W_{\bar{q} q \beta \gamma}(\omega) \Gamma_{\bar{q} q \sigma \gamma \delta}^{b}(\omega,-k, k-\omega) W_{\sigma \sigma}^{a b}(k-\omega)\right. \\
& \left.+\Gamma_{\bar{q} q A \alpha \beta i}^{(0) a}(k,-\omega, \omega-k) W_{\bar{q} q \beta \gamma}(\omega) \Gamma_{\bar{q} q A \gamma \delta j}^{b}(\omega,-k, k-\omega) W_{A A i j}^{a b}(k-\omega)\right\}
\end{aligned}
$$

( $W_{A A}$ is the spatial gluon propagator, which will be unimportant here). The full quark-gluon vertices obey the Slavnov-Taylor identity. The derivation of this identity follows from the invariance of the action under a Gauss-BRST transform [24] that is peculiar to Coulomb gauge and is similar to the identities derived for the pure Yang-Mills sector [29]. This derivation is presented in Appendix A (and is a technical result of this study in its own right). The identity reads, Eq. (A.18):

$$
\begin{aligned}
k_{3}^{0} \Gamma_{\bar{q} q \sigma \alpha \beta}^{d}\left(k_{1}, k_{2}, k_{3}\right)= & \imath \frac{k_{3 i}}{\vec{k}_{3}^{2}} \Gamma_{\bar{q} q A \alpha \beta i}^{a}\left(k_{1}, k_{2}, k_{3}\right) \Gamma_{\bar{c} c}^{a d}\left(-k_{3}\right) \\
& +\Gamma_{\bar{q} q \alpha \delta}\left(k_{1}\right)\left[\tilde{\Gamma}_{\bar{q} ; \bar{c} c q}^{d}\left(k_{1}+q_{0}, k_{3}-q_{0} ; k_{2}\right)+\imath g T^{d}\right]_{\delta \beta} \\
& +\left[\tilde{\Gamma}_{q ; \bar{c} c \bar{q}}^{d}\left(k_{2}+q_{0}, k_{3}-q_{0} ; k_{1}\right)-\imath g T^{d}\right]_{\alpha \delta} \Gamma_{\bar{q} q \delta \beta}\left(-k_{2}\right)
\end{aligned}
$$

where $k_{1}+k_{2}+k_{3}=0, q_{0}$ is the arbitrary energy injection scale, $\Gamma_{\bar{c} c}$ is the ghost proper two-point function, $\tilde{\Gamma}_{\bar{q} ; \bar{c} c q}$ and $\tilde{\Gamma}_{q ; \bar{c} c \bar{q}}$ are ghost-quark kernels associated with the Gauss-BRST transform (see Appendix for details).

In order to use the Slavnov-Taylor identity as input for solving the gap equation, we first apply our truncation scheme in the context of the heavy mass expansion at leading order. Starting with the dressed spatial quark-gluon vertex, consider the terms that contribute to the Dyson-Schwinger equation shown schematically in Fig. 1. According to the truncation scheme, we set all Yang-Mills vertices to zero, meaning that diagrams (c-f) are excluded. This then leaves us with the tree-level term (a) and the quark-loop term (b). However, both of these involve at least one tree-level spatial quark-gluon vertex which is not present at leading order in the mass expansion. Thus, we obtain the nonperturbative result that

$$
\Gamma_{\bar{q} q A \beta i}^{a}\left(k_{1}, k_{2}, k_{3}\right)=\mathcal{O}(1 / m) .
$$

It is straightforward to justify that the ghost-quark kernels of the Slavnov-Taylor identity, given their definition, Eq. (A.19), involve Yang-Mills vertices and do not contribute here. Thus, in our truncation scheme and at leading order in the mass expansion, the Slavnov-Taylor identity reads

$$
k_{3}^{0} \Gamma_{\bar{q} q \sigma \alpha \beta}^{d}\left(k_{1}, k_{2}, k_{3}\right)=\Gamma_{\bar{q} q \alpha \delta}\left(k_{1}\right)\left[\imath g T^{d}\right]_{\delta \beta}-\left[\imath g T^{d}\right]_{\alpha \delta} \Gamma_{\bar{q} q \delta \beta}\left(-k_{2}\right)+\mathcal{O}(1 / m) .
$$

Clearly, the truncation scheme thus results in an Abelian type Ward identity. Moreover, since the temporal quarkgluon vertex is simply multiplied by the temporal gluon energy (the primary feature of Coulomb, as opposed to 


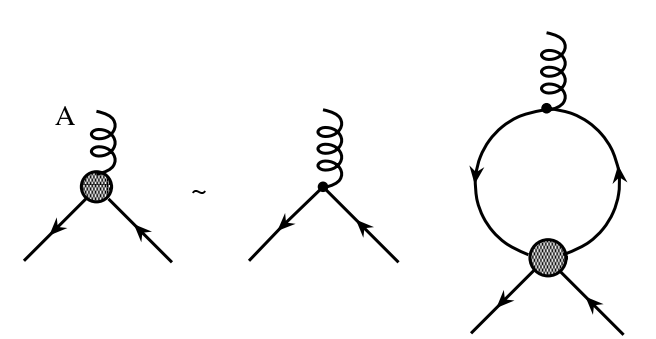

(a)

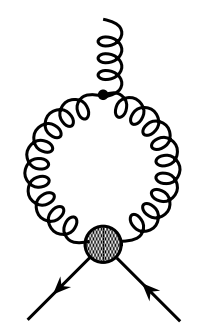

(c)

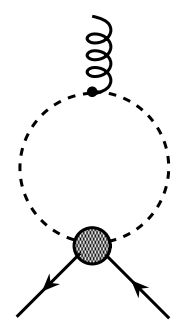

(d)

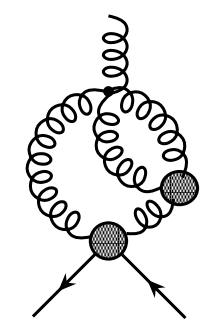

(e)

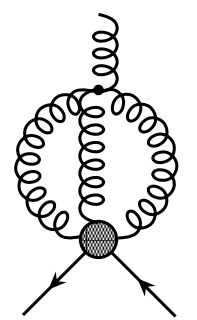

(f)

FIG. 1: Diagrams that contribute to the Dyson-Schwinger equation for the spatial quark-gluon vertex (without prefactors or signs). Internal propagators are fully dressed and blobs represent dressed proper vertex and (reducible) kernels. Internal propagators represented by springs may be either spatial $(\vec{A})$ or temporal $(\sigma)$ propagators, dashed lines represent the ghost propagator and solid lines represent the quark propagator.

covariant, gauge Slavnov-Taylor identities) and the quark proper two-point function is color diagonal, we can immediately write the solution:

$$
\Gamma_{\bar{q} q \sigma \alpha \beta}^{d}\left(k_{1}, k_{2}, k_{3}\right)=\frac{\imath g}{k_{3}^{0}}\left\{T^{d}\left[\Gamma_{\bar{q} q}\left(k_{1}\right)-\Gamma_{\bar{q} q}\left(-k_{2}\right)\right]\right\}_{\alpha \beta}+\mathcal{O}(1 / m) .
$$

The above solution is trivially satisfied at tree-level. We notice however, that there appears to be a potential problem with the energy. When $k_{3}^{0}=0$, but $\vec{k}_{3} \neq 0$ (a spacelike gluon configuration), the simple pole must somehow be canceled by the difference of proper quark two-point functions. Since the spatial momentum configuration is arbitrary, this means that $\Gamma_{\bar{q} q}(k) \rightarrow \Gamma_{\bar{q} q}\left(k_{0}\right)+\mathcal{O}(1 / m)$. The demand that the nonperturbative vertex solution to the Coulomb gauge Slavnov-Taylor identity be free of kinematic divergences (here, simply the $1 / k_{3}^{0}$ factor) is a variation of the familiar covariant gauge situation considered in Ref. [38].

Inserting the results, Eq. (3.5) and Eq. (3.3), for the vertices, using the Feynman rules given by Eq. (2.30), with the temporal gluon propagator given by Eq. (2.32) and resolving the color structure the nonperturbative gap equation, Eq. (3.1) under truncation and at leading order in the mass expansion thus reads

$$
\Gamma_{\bar{q} q \alpha \delta}\left(k_{0}\right)=\imath\left[k_{0}-m\right] \delta_{\alpha \delta}-g^{2} C_{F} \int \frac{d \omega D_{\sigma \sigma}(\vec{k}-\vec{\omega})}{\left(k_{0}-\omega_{0}\right)(\vec{k}-\vec{\omega})^{2}} W_{\bar{q} q \alpha \beta}\left(\omega_{0}\right)\left[\Gamma_{\bar{q} q}\left(\omega_{0}\right)-\Gamma_{\bar{q} q}\left(k_{0}\right)\right]_{\beta \delta}+\mathcal{O}(1 / m) .
$$

There exists one particularly simple solution to this equation as we shall now demonstrate. It is given by

$$
W_{\bar{q} q \alpha \beta}(k)=\frac{-\imath \delta_{\alpha \beta}}{\left[k_{0}-\mathcal{C}+\imath \varepsilon\right]}+\mathcal{O}(1 / m), \quad \Gamma_{\bar{q} q \alpha \beta}(k)=\imath \delta_{\alpha \beta}\left[k_{0}-\mathcal{C}\right]+\mathcal{O}(1 / m)
$$

and where the constant $\left(d \vec{\omega}=d^{3} \vec{\omega} /(2 \pi)^{3}\right)$

$$
\mathcal{C}=m+\frac{1}{2} g^{2} C_{F} \int \frac{d \vec{\omega} D_{\sigma \sigma}(\vec{\omega})}{\vec{\omega}^{2}}+\mathcal{O}(1 / m) .
$$

Putting the above solution into the Slavnov-Taylor identity, we also have that for the vertex

$$
\Gamma_{\bar{q} q \sigma \alpha \beta}^{d}\left(k_{1}, k_{2}, k_{3}\right)=\left[g T^{d}\right]_{\alpha \beta}+\mathcal{O}(1 / m) .
$$


In other words, the dressed temporal quark-gluon vertex is trivial and the gap equation reduces to the rainbow truncation. There is a subtlety to the solution, Eq. (3.7), involving the ordering of the limits in the spatial and temporal integrals and potential divergences in the constant given by Eq. (3.8). Here, let us consider the case when we perform the temporal integral first under the condition that the spatial integral is somehow regularized and finite (this will be done throughout the rest of the paper). Inserting the solution given by Eq. (3.7), the gap equation under truncation, Eq. (3.6), and with a regularized (i.e., finite) spatial integral denoted by the subscript $r$, reads

$$
\begin{aligned}
\mathcal{C}_{r} & =m+g^{2} C_{F} \int_{r} \frac{d \vec{\omega} D_{\sigma \sigma}(\vec{k}-\vec{\omega})}{(\vec{k}-\vec{\omega})^{2}} \frac{\imath}{2 \pi} \lim _{R \rightarrow \infty} \int_{-R}^{R} \frac{d \omega_{0}}{\left[\omega_{0}-\mathcal{C}_{r}+\imath \varepsilon\right]}+\mathcal{O}(1 / m) \\
& =m+\frac{1}{2} g^{2} C_{F} \int_{r} \frac{d \vec{\omega} D_{\sigma \sigma}(\vec{k}-\vec{\omega})}{(\vec{k}-\vec{\omega})^{2}}+\mathcal{O}(1 / m) .
\end{aligned}
$$

The effect of performing the temporal integral first is that the regularized constant $\mathcal{C}_{r}$ in the denominator factor becomes irrelevant. The spatial integral now involves no external scale and removing the spatial regularization, we arrive at our above result, Eq. (3.8).

A brief discussion about the physical interpretation of these results is in order. Firstly, it might be the case that there exist other solutions to the truncated gap equation. As will be shown in the next Section though, the above solution can also be derived from a semi-perturbative type of expansion. However, as is the case with many systems, especially those with strong couplings or phase transitions, the fully nonperturbative solution might not be the same as the resummed perturbative solution. Second, that the solution involves potentially divergent constants is not a comfortable situation but does not necessarily contradict the physics. We notice that the quark propagator has a single pole, so cannot represent physical propagation (which requires a covariant double pole) and this arises obviously from the truncation of the mass expansion and where the charge conjugation symmetry has been explicitly broken. Indeed, the position of the pole has no physical meaning since the quark can never be on-shell. That the single pole is shifted to infinity simply means that either one requires infinite energy to create a quark from the vacuum or that should one have an incoming quark (from some other hadron), only the relative energy is important. Note that in Coulomb gauge it is known that the total color charge of the system is conserved and vanishing [25], so one cannot prepare an isolated colored state of a single quark under any circumstances. Further, the divergences here have no interpretation with regards to renormalization, at least within the context of the mass expansion to leading order. The mass parameter cannot be renormalized simply because one cannot construct an appropriate counterterm in the action that is linear in $m$ to absorb the constant $\mathcal{C}$. Also, the quark field renormalization is trivial at leading order, as one sees from the explicit form of the temporal quark-gluon vertex, Eq. (3.9). The upshot of this is that one must consider only the relative energies in the system - the divergence of the absolute energy has no physical meaning and this will become especially relevant when we study the Bethe-Salpeter equation (indeed this has been known for quite some time, see for example Ref. [19]).

Having discussed the quark propagator, let us now discuss the antiquark propagator. Recall that at tree-level, we used a different Feynman prescription for the two denominator factors and this gives rise to some rather interesting physical consequences. As previously discussed, the heavy mass expansion employed here breaks the charge conjugation symmetry relating particle and antiparticle, so we cannot expect that the two propagators are necessarily equivalent. Starting with the gap equation for full QCD, Eq. (3.1), we reverse the ordering of the quark and antiquark functional derivatives that form the quark Green's functions (still within the context of the full quark fields and sources) and rearrange the ordering to get the gap equation for the antiquark propagator:

$$
\begin{aligned}
-\Gamma_{q \bar{q} \delta \alpha}(-k)=-\Gamma_{q \bar{q} \delta \alpha}^{(0)}(-k)-\int d \omega & \left\{\Gamma_{q \bar{q} \sigma \delta \gamma}^{b}(-k, \omega, k-\omega) W_{q \bar{q} \gamma \beta}(-\omega) \Gamma_{q \bar{q} \sigma \beta \alpha}^{(0) a}(-\omega, k, \omega-k) W_{\sigma \sigma}^{a b}(k-\omega)\right. \\
& \left.+\Gamma_{q \bar{q} A \delta \gamma j}^{b}(-k, \omega, k-\omega) W_{q \bar{q} \gamma \beta}(-\omega) \Gamma_{q \bar{q} A \beta \alpha i}^{(0) a}(-\omega, k, \omega-k) W_{A A i j}^{a b}(k-\omega)\right\} .
\end{aligned}
$$

Applying our truncation scheme reduces the above to

$$
\Gamma_{q \bar{q} \delta \alpha}(-k)=\Gamma_{q \bar{q} \delta \alpha}^{(0)}(-k)+\int d \omega \Gamma_{q \bar{q} \sigma \delta \gamma}^{b}(-k, \omega, k-\omega) W_{q \bar{q} \gamma \beta}(-\omega) \Gamma_{q \bar{q} \sigma \beta \alpha}^{(0) a}(-\omega, k, \omega-k) W_{\sigma \sigma}^{a b}(k-\omega)+\mathcal{O}(1 / m) .
$$

In similar fashion, we have the Slavnov-Taylor identity for the antiquark-gluon vertex:

$$
-k_{3}^{0} \Gamma_{q \bar{q} \sigma \beta \alpha}^{d}\left(k_{2}, k_{1}, k_{3}\right)=+\Gamma_{q \bar{q} \beta \delta}\left(k_{2}\right)\left[i g T^{d}\right]_{\delta \alpha}^{T}-\left[i g T^{d}\right]_{\beta \delta}^{T} \Gamma_{q \bar{q} \delta \alpha}\left(-k_{1}\right)+\mathcal{O}(1 / m) .
$$




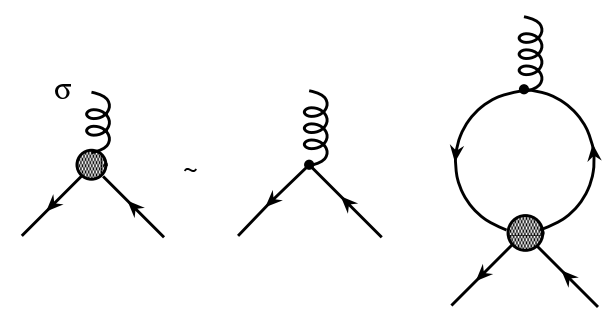

(a)

(b)

FIG. 2: Diagrams that contribute under our truncation scheme to the Dyson-Schwinger equation for the temporal quark-gluon vertex (without prefactors or signs). Internal propagators are fully dressed and blobs represent dressed proper vertex and (reducible) kernels. Internal propagators represented by solid lines represent the quark propagator.

The form of the solution to Eq. (3.12) is similar to the previous results:

$$
W_{q \bar{q} \alpha \beta}(k)=\frac{-\imath \delta_{\alpha \beta}}{\left[k_{0}+\overline{\mathcal{C}}_{r}+\imath \varepsilon\right]}+\mathcal{O}(1 / m), \quad \Gamma_{q \bar{q} \alpha \beta}(k)=\imath \delta_{\alpha \beta}\left[k_{0}+\overline{\mathcal{C}}_{r}\right]+\mathcal{O}(1 / m)
$$

with the corresponding solution for the vertex

$$
\Gamma_{q \bar{q} \sigma \alpha \beta}^{d}\left(k_{1}, k_{2}, k_{3}\right)=-\left[g T^{d}\right]_{\beta \alpha}+\mathcal{O}(1 / m) .
$$

However, the constant (obtained in the same way as before by first doing the temporal integral under the assumption that the spatial integral is regularized in some hypothetical manner) is now

$$
\overline{\mathcal{C}}_{r}=m-\frac{1}{2} g^{2} C_{F} \int_{r} \frac{d \vec{\omega} D_{\sigma \sigma}(\vec{\omega})}{\vec{\omega}^{2}}+\mathcal{O}(1 / m)
$$

We notice that the sign of the loop correction has changed and this will turn out (in the context of the Bethe-Salpeter equations for mesons and diquarks) to have far-reaching consequences. For the moment we interpret this result as being simply another manifestation of the breaking of the charge conjugation (particle-antiparticle) symmetry via the mass expansion.

\section{GAP EQUATION: SEMIPERTURBATIVE TREATMENT}

Whilst we have already solved the gap (and anti-gap) equation, it proves instructive to reconsider it within the context of a semiperturbative analysis since this will naturally introduce a technical feature crucial for considering the Bethe-Salpeter equation nonperturbatively. We have seen that the solutions for the proper two-point function leads to a temporal quark-gluon (and antiquark-gluon) vertex that is not dressed. Under our truncation scheme, the nonperturbative Dyson-Schwinger equation for the temporal quark-gluon vertex involves the diagrams shown in Fig. 2. The semiperturbative expansion is based on a hybrid loop expansion whereby all internal propagators are taken to be dressed, but all internal vertices are tree-level. To show that all the loop corrections (contained in diagram (b) of Fig. 2) vanish, it suffices to consider two types of diagram, given in Figs. 3 and 4.

In Fig. 3 we consider a single ladder exchange correction to the temporal quark-gluon vertex. This diagram (neglecting the overall color and prefactors) gives rise to the following scalar integral (as in the previous section, the spatial integral is regularized such that one can perform the temporal integral first):

$$
\int_{r} \frac{d \vec{\omega} D_{\sigma \sigma}(\vec{k}-\vec{\omega})}{(\vec{k}-\vec{\omega})^{2}} \frac{1}{2 \pi} \int_{-\infty}^{\infty} \frac{d \omega_{0}}{\left[\omega_{0}-\mathcal{C}_{r}+\imath \varepsilon\right]\left[\omega_{0}+q_{0}-\mathcal{C}_{r}+\imath \varepsilon\right]} .
$$

Now we apply the following identity (for finite, real $a, b$; the case $a=b$ is trivial):

$$
\int_{-\infty}^{\infty} \frac{d z}{[z-a+\imath \varepsilon][z-b+\imath \varepsilon]}=\frac{1}{(a-b)} \int_{-\infty}^{\infty} d z\left\{\frac{1}{[z-a+\imath \varepsilon]}-\frac{1}{[z-b+\imath \varepsilon]}\right\}=0 .
$$




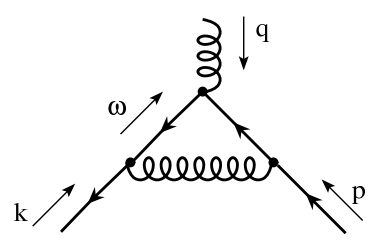

FIG. 3: Ladder type loop correction to the temporal quark-gluon vertex. Internal propagators are fully dressed: solid lines represent the quark propagator and springs denote the temporal gluon propagator.

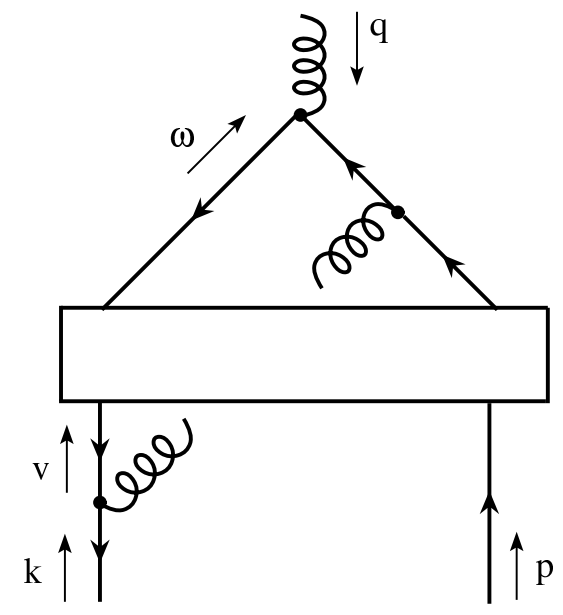

FIG. 4: Generic crossed box type loop correction to the temporal quark-gluon vertex. Internal propagators are fully dressed: solid lines represent the quark propagator and springs denote the temporal gluon propagator. The box represents any combination of interactions allowed under our truncation.

Thus we see that where the spatial integral is regularized, the temporal integral vanishes. It is simple to see that the planar one-loop diagrams with two or more external temporal gluon legs (which under the truncation scheme considered here connect only to the internal quark line) and one internal temporal gluon will also vanish.

Now let us consider a generic crossed box (nonplanar) type of diagram, illustrated in Fig. 4. Considering only the temporal double integral components of the explicit internal quark propagators, we have the following form

$$
\begin{aligned}
& \int_{-\infty}^{\infty} \frac{d \omega_{0} d v_{0}}{\left[v_{0}-a_{1}+\imath \varepsilon\right]\left[\omega_{0}-a_{2}+\imath \varepsilon\right]\left[\omega_{0}+q_{0}-a_{3}+\imath \varepsilon\right]\left[\omega_{0}-v_{0}-p_{0}-a_{4}+\imath \varepsilon\right]} \\
& =\int_{-\infty}^{\infty} \frac{d \omega_{0}}{\left[\omega_{0}-a_{2}+\imath \varepsilon\right]\left[\omega_{0}+q_{0}-a_{3}+\imath \varepsilon\right]\left[\omega_{0}-p_{0}-a_{1}-a_{4}+2 \imath \varepsilon\right]} \\
& \times \int_{-\infty}^{\infty} d v_{0}\left\{\frac{1}{\left[v_{0}-a_{1}+\imath \varepsilon\right]}-\frac{1}{\left[v_{0}-\omega_{0}+p_{0}+a_{4}-\imath \varepsilon\right]}\right\} \\
& =-2 \pi \imath \int_{-\infty}^{\infty} \frac{d \omega_{0}}{\left[\omega_{0}-a_{2}+\imath \varepsilon\right]\left[\omega_{0}+q_{0}-a_{3}+\imath \varepsilon\right]\left[\omega_{0}-p_{0}-a_{1}-a_{4}+2 \imath \varepsilon\right]} \\
& =0
\end{aligned}
$$

where in the last line, we have used a variation of the identity Eq. (4.2). Thus we have the result that the generic crossed box type of diagram shown in Fig. 4 also vanishes.

Given that both the single ladder type exchange diagram and the generic crossed box diagrams considered so far vanish, it is easy to see that any vertex dressing diagram will vanish (including all subdiagrams such as internal vertex corrections and so on), since all diagrams are merely variations or combinations of these two under our truncation scheme. This result is a consequence of the fact that the energy and Feynman prescription of the denominator factors follow the quark line through the diagram so that eventually the identity, Eq. (4.2), can be used. It is also precisely the reason why all closed quark loops vanish, as previously discussed. 


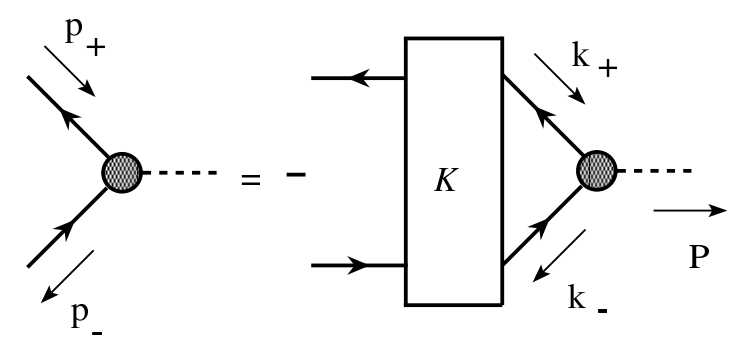

FIG. 5: Homogeneous Bethe-Salpeter equation for quark-antiquark bound states. Internal propagators are fully dressed and solid lines represent the quark propagator. The box represents the Bethe-Salpeter kernel $K$ and filled blobs represent the Bethe-Salpeter vertex function $\Gamma$ with the (external) bound state leg given by a dashed line. See text for details.

Thus, the semiperturbative expansion confirms our previous result that the temporal quark-gluon vertex remains bare to all orders. Also clear should be that the result applies to the antiquark-gluon vertex too. With the corresponding simple forms for the self-energy integrals of the gap and anti-gap equations (which as we recall, reduce to the rainbow truncation), the results for the quark propagator functions are also confirmed. Notice though that whilst the all orders semiperturbative result must match the nonperturbative result, the converse is not necessarily true. It remains the case that there may exist further solutions, but these must be purely nonperturbative in character if they exist. That the identities that have been introduced here conform to the nonperturbative case previously studied is useful since it allows us to apply them with confidence to the Bethe-Salpeter equation in the next Sections.

\section{BETHE-SALPETER EQUATION: MESONS}

As has been emphasized, because we initially consider the generating functional for full quark sources and fields we are at liberty to use the full functional (nonperturbative) equations as a starting point and then subsequently apply our mass expansion and truncation scheme. Let us now consider the homogeneous Bethe-Salpeter equation for quark-antiquark bound states. In full QCD and with our conventions (the minus sign arises from the definitions of the Legendre transform and Green's functions), this equation reads

$$
\Gamma_{\alpha \beta}(p ; P)=-\int d k K_{\alpha \beta ; \delta \gamma}(p, k ; P)\left[W_{\bar{q} q}\left(k_{+}\right) \Gamma(k ; P) W_{\bar{q} q}\left(k_{-}\right)\right]_{\gamma \delta}
$$

where $k_{+}=k+\xi P, k_{-}=k+(\xi-1) P$ (similarly for $p_{ \pm}$) are the momenta of the quarks, $P$ is the pole 4-momentum of the bound state (assuming that a solution exists), $\xi=[0,1]$ is the so-called momentum sharing fraction that dictates how much of the total meson momentum is carried by each quark constituent, $K$ represents the Bethe-Salpeter kernel and $\Gamma$ is the Bethe-Salpeter vertex function for the particular bound state that one is considering and whose indices explicitly denote only its quark content (we will see later what color, flavor and spin structure the solutions may have). Physically, results should be independent of $\xi$ and this has been numerically observed in phenomenological studies [7]. The Bethe-Salpeter equation is shown pictorially in Fig. 5.

Aside from the quark propagators (which we shall discuss shortly), the central element to solving the Bethe-Salpeter equation involves the construction of the kernel $K$. As discussed in the Introduction, for technical reasons the most widely studied system is based on the ladder kernel which is either constructed via the interchange of a single gluon (for example [8]) or as a phenomenological potential (see for example Ref. [19]). However, there has been much recent attention focused on the construction of more sophisticated kernels. One key element of the construction is the axialvector Ward-Takahashi identity [AXWTI], which relates the gap equation to the Bethe-Salpeter kernel and which ensures that chiral symmetry and its spontaneous breaking are consistently implemented (e.g., Refs. [10, 15, 19]). Here, we shall show that the ladder Bethe-Salpeter kernel is exact at leading order in the heavy mass expansion and under our truncation scheme. This derivation follows in the same way as the semiperturbative analysis of the previous Section.

Following from the truncation of the heavy mass expansion to leading order, it was argued that the antiquark propagator must be treated as distinct from the quark propagator. This means that we have to be very explicit about which propagator is which. Since we are studying the quark-antiquark system (we will analyze the quark-quark, or diquark system in the next Section), the Bethe-Salpeter equation for our purposes more properly reads

$$
\Gamma_{\alpha \beta}(p ; P)=-\int d k K_{\alpha \beta ; \delta \gamma}(p, k ; P)\left[W_{\bar{q} q}\left(k_{+}\right) \Gamma(k ; P)(-1) W_{q \bar{q}}^{T}\left(-k_{-}\right)\right]_{\gamma \delta}
$$




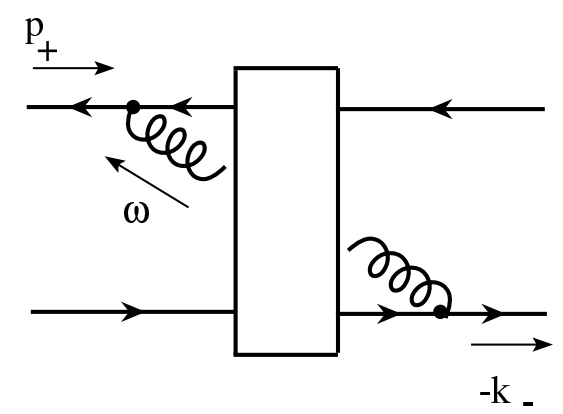

FIG. 6: Generic crossed box type of diagram that contributes to the Bethe-Salpeter kernel. Internal propagators are fully dressed, whereas vertices are tree-level. The upper (lower) solid line denotes the quark (antiquark) propagator; springs denote the temporal gluon propagator and the box represents any combination of nontrivial interactions allowed under our truncation scheme. See text for details.

where we have explicitly identified the antiquark propagator contribution (it corresponds to the lower line of Fig. 5) by reordering the functional derivatives and there is implicitly a similar antiquark contribution within the kernel which absorbs the explicit minus sign. Recall that we are implicitly considering only the flavor non-singlet case.

To construct the full Bethe-Salpeter kernel, consider the generic semiperturbative crossed box contribution given in Fig. 6. As before, at leading order in the mass expansion and under our truncation scheme we only have the temporal quark-gluon and antiquark-gluon vertices, both of which have been shown to be given by their tree-level forms. Additionally, it was justified that all planar diagrams with multiple external temporal gluon legs vanish. Thus, aside from the ladder contribution to the Bethe-Salpeter kernel, this generic crossed box diagram contains all possible nontrivial contributions. Such a diagram has at least the following terms in the temporal integral (as before, we assume that the spatial integral is regularized and finite so that we are able to firstly perform the temporal integral without complication)

$$
\int \frac{d \omega_{0}}{\left[\omega_{0}+p_{+}^{0}-\mathcal{C}_{r}+\imath \varepsilon\right] \ldots\left[\omega_{0}-k_{-}^{0}+\overline{\mathcal{C}}_{r}+\imath \varepsilon\right]}
$$

The first factor corresponds to the explicit quark (upper) propagator, the last factor to the explicit antiquark (lower) propagator. Implicitly (represented by the dots), there may be multiple propagator factors which carry the same dependence on the integration energy $\omega_{0}$ but crucially, all these will have the same relative sign for the Feynman prescription term, i.e., $\omega_{0}+\imath \varepsilon$, regardless of whether they originate from internal quark or antiquark propagators. Therefore, this type of integral can always be reduced to the difference of integrals over a simple pole and with the same sign for carrying out the analytic integration, just as in Eq. (4.2) and the semiperturbative treatment of the vertex discussed in the last Section. Thus, all generic crossed box diagrams in the Bethe-Salpeter kernel are zero and one is left with simply the ladder contribution to the kernel. Actually, this could have been anticipated from the beginning - the AXWTI connects the self-energy term of the gap equation and the Bethe-Salpeter kernel and since it has been shown explicitly that the self-energy integral reduces to the rainbow truncation, the corresponding Bethe-Salpeter kernel is simply given by ladder exchange.

Given all this, the Bethe-Salpeter equation for the quark-antiquark system, at leading order in the mass expansion and within our truncation scheme, can be explicitly written as

$$
\begin{aligned}
\Gamma_{\alpha \beta}(p ; P)= & -\int d k \Gamma_{\bar{q} q \sigma \alpha \gamma}^{a}\left(p_{+},-k_{+}, k-p\right) W_{\sigma \sigma}^{a b}(p-k) \Gamma_{q \bar{q} \sigma \beta \delta}^{b T}\left(-p_{-}, k_{-}, p-k\right) W_{\bar{q} q \gamma \lambda}\left(k_{+}\right) W_{q \bar{q} \delta \kappa}^{T}\left(-k_{-}\right) \Gamma_{\lambda \kappa}(k ; P) \\
& +\mathcal{O}(1 / m) .
\end{aligned}
$$

Inserting the nonperturbative results for the propagators and vertices so far, Eqs. $(3.7,3.14,3.9,3.15)$ and taking the form, Eq. (2.32), for the temporal gluon propagator, we get the equation (the temporal and spatial integrals are separated as before)

$$
\Gamma_{\alpha \beta}(p ; P)=g^{2} \int_{r} \frac{d \vec{k} D_{\sigma \sigma}(\vec{p}-\vec{k})}{(\vec{p}-\vec{k})^{2}} \frac{\imath}{2 \pi} \int_{-\infty}^{\infty} \frac{d k_{0}}{\left[k_{+}^{0}-\mathcal{C}_{r}+\imath \varepsilon\right]\left[k_{-}^{0}-\overline{\mathcal{C}}_{r}-\imath \varepsilon\right]}\left[T^{a} \Gamma(k ; P) T^{a}\right]_{\alpha \beta}+\mathcal{O}(1 / m) .
$$

We see immediately that the flavor and spin structure of the meson decouples from the problem - this is well a known property of the heavy mass expansion. The color structure will be discussed shortly. Since the external energy $p_{0}$ 
does not enter the right-hand side of the above equation, we further have that the Bethe-Salpeter equation must be independent of the relative quark energy (and only implicitly dependent on the putative bound state energy $P_{0}$ ) . Thus we can write

$$
\begin{aligned}
\Gamma_{\alpha \beta}(\vec{p} ; P) & =g^{2} \int_{r} \frac{d \vec{k} D_{\sigma \sigma}(\vec{p}-\vec{k})}{(\vec{p}-\vec{k})^{2}}\left[T^{a} \Gamma(\vec{k} ; P) T^{a}\right]_{\alpha \beta} \frac{\imath}{2 \pi} \int_{-\infty}^{\infty} \frac{d k_{0}}{\left[k_{+}^{0}-\mathcal{C}_{r}+\imath \varepsilon\right]\left[k_{-}^{0}-\overline{\mathcal{C}}_{r}-\imath \varepsilon\right]}+\mathcal{O}(1 / m) \\
& =-g^{2} \int_{r} \frac{d \vec{k} D_{\sigma \sigma}(\vec{p}-\vec{k})}{(\vec{p}-\vec{k})^{2}} \frac{\left[T^{a} \Gamma(\vec{k} ; P) T^{a}\right]_{\alpha \beta}}{\left[P_{0}-\mathcal{C}_{r}+\overline{\mathcal{C}}_{r}+2 \imath \varepsilon\right]}+\mathcal{O}(1 / m) .
\end{aligned}
$$

Thus, at leading order in the mass expansion, inserting the expressions Eq. (3.10) and Eq. (3.16), for $\mathcal{C}_{r}$ and $\overline{\mathcal{C}}_{r}$, respectively, it is now clear that

$$
\left[P_{0}-g^{2} C_{F} \int_{r} \frac{d \vec{\omega} D_{\sigma \sigma}(\vec{\omega})}{\vec{\omega}^{2}}\right] \Gamma_{\alpha \beta}(\vec{p} ; P)=-g^{2} \int_{r} \frac{d \vec{k} D_{\sigma \sigma}(\vec{p}-\vec{k})}{(\vec{p}-\vec{k})^{2}}\left[T^{a} \Gamma(\vec{k} ; P) T^{a}\right]_{\alpha \beta}+\mathcal{O}(1 / m) .
$$

We notice that the explicit quark mass contributions of the self-energy expressions cancel. This is a feature of the quark-antiquark Bethe-Salpeter equation - it does not make any reference to the origins of its constituents and why for example the pion can be a massless bound state of massive constituents. Physically, one can visualize that the quark and antiquark are moving with equal and opposite velocities such that the centre of mass system (the bound state) is stationary. This is related explicitly to the choice of Feynman prescription for the constituent quark and antiquark. Were the Feynman prescription for the antiquark chosen to coincide with that of the quark, the right-hand side of Eq. (5.6) would simply vanish and there would be certainly no physical quark-antiquark state. The Feynman prescription for the antiquark corresponds precisely to a particle moving with the opposite velocity. Also, at leading order in the mass expansion, the momentum sharing parameter, $\xi$, has dropped out. Thus, the results retain the important physical requirement that they be independent of $\xi$. Shifting integration momenta, we can write

$$
P_{0} \Gamma_{\alpha \beta}(\vec{p} ; P)=g^{2} \int_{r} \frac{d \vec{\omega} D_{\sigma \sigma}(\vec{\omega})}{\vec{\omega}^{2}}\left\{C_{F} \Gamma_{\alpha \beta}(\vec{p} ; P)-\left[T^{a} \Gamma(\vec{p}-\vec{\omega} ; P) T^{a}\right]_{\alpha \beta}\right\}+\mathcal{O}(1 / m) .
$$

To see the physical meaning of this equation, we rewrite the Bethe-Salpeter vertex function as a Fourier transform:

$$
\Gamma_{\alpha \beta}(\vec{p} ; P)=\int d \vec{y} e^{-\imath \vec{p} \cdot \vec{y}} \Gamma_{\alpha \beta}(\vec{y})
$$

(in the homogeneous Bethe-Salpeter equation, the total momentum $P$ denotes the solution and is not a variable). We also write for the color structure

$$
\left[T^{a} \Gamma(\vec{y}) T^{a}\right]_{\alpha \beta}=C_{M} \Gamma_{\alpha \beta}(\vec{y})
$$

where $C_{M}$ is yet to be identified. Then, the Bethe-Salpeter equation reduces to

$$
\int d \vec{y} e^{-\imath \vec{p} \cdot \vec{y}} P_{0} \Gamma_{\alpha \beta}(\vec{y})=\int d \vec{y} e^{-\imath \vec{p} \cdot \vec{y}} g^{2} \int_{r} \frac{d \vec{\omega} D_{\sigma \sigma}(\vec{\omega})}{\vec{\omega}^{2}}\left\{C_{F} \Gamma_{\alpha \beta}(\vec{y})-e^{\imath \vec{\omega} \cdot \vec{y}} C_{M} \Gamma_{\alpha \beta}(\vec{y})\right\}+\mathcal{O}(1 / m)
$$

with the simple solution

$$
P_{0}=g^{2} \int_{r} \frac{d \vec{\omega} D_{\sigma \sigma}(\vec{\omega})}{\vec{\omega}^{2}}\left\{C_{F}-e^{\imath \vec{\omega} \cdot \vec{y}} C_{M}\right\}+\mathcal{O}(1 / m) .
$$

Because the total color charge of the system is conserved and vanishing [25], neither the quark or antiquark can exist as an independent asymptotic physical state. Thus, the bound state energy, $P_{0}$, can only increase linearly as the separation between the them increases (physically confining) or be infinite when the hypothetical regularization is removed (so that the system cannot be physically created). Whether the system is confining or disallowed can only depend on the color structure, since the temporal gluon propagator dressing function would be common to both situations. In configuration space, an infrared confining solution is characterized by the solution $P_{0}=\sigma|\vec{y}|$ for large $|\vec{y}|$ and where $\sigma$ is the string tension such that as the separation between the quark and antiquark increases, the energy of the system should increase linearly without bound and infinite energy input is required to fully separate them (at least in the absence of unquenching). The small $|\vec{y}|$ (and large $|\vec{\omega}|)$ properties are of no concern here. The Fourier transform integral needed for the infrared confining solution is

$$
\int \frac{d \vec{\omega}}{\vec{\omega}^{4}}\left[1-e^{\imath \vec{\omega} \cdot \vec{y}}\right]=\frac{|\vec{y}|}{8 \pi} .
$$


If the temporal gluon propagator dressing function is more infrared divergent than $1 /|\vec{\omega}|$, as above, then

$$
C_{F}=C_{M}
$$

is required such that the spatial integral is convergent and the energy of the system well-defined, as the hypothetical regularization is removed (since we are interested in the low $|\vec{\omega}|$ regime, it becomes clear that the regularization here would be infrared in character). Using the Fierz identity for the generators $T^{a}$ :

$$
2\left[T^{a}\right]_{\alpha \beta}\left[T^{a}\right]_{\delta \gamma}=\delta_{\alpha \gamma} \delta_{\delta \beta}-\frac{1}{N_{c}} \delta_{\alpha \beta} \delta_{\delta \gamma}
$$

gives the condition

$$
C_{F} \Gamma_{\alpha \gamma}(\vec{y}) \equiv C_{M} \Gamma_{\alpha \gamma}(\vec{y})=\frac{1}{2} \delta_{\alpha \gamma} \Gamma_{\beta \beta}(\vec{y})-\frac{1}{2 N_{c}} \Gamma_{\alpha \gamma}(\vec{y}),
$$

or with the definition Eq. (2.3),

$$
\Gamma_{\alpha \gamma}(\vec{y})=\delta_{\alpha \gamma} \Gamma(\vec{y}) .
$$

In other words, the quark-antiquark Bethe-Salpeter equation with $D_{\sigma \sigma}$ more infrared singular than $1 /|\vec{\omega}|$ can only have a finite solution for color singlet states where the divergent constant integral coming from the unphysical quark self-energy cancels; otherwise the energy of the system is divergent.

Another way to see that only color-singlet states are physical is to consider that if the temporal gluon propagator is the origin of a potential in configuration space, this potential can always be shifted by some spatial constant [19]. In momentum space, this means that one can make the replacement

$$
g^{2} \frac{D_{\sigma \sigma}(\vec{\omega})}{\vec{\omega}^{2}} \rightarrow g^{2} \frac{D_{\sigma \sigma}(\vec{\omega})}{\vec{\omega}^{2}}+\text { const } \times(2 \pi)^{3} \delta(\vec{\omega})
$$

without changing the physical bound state energy. This automatically gives the constraint $C_{F}=C_{M}$ regardless of whether or not the temporal gluon propagator is infrared enhanced.

Assuming that in the infrared (as is indicated by the lattice data [32] or by the above argument about the nonexistence of asymptotic quark states), $D_{\sigma \sigma}=X / \vec{\omega}^{2}$ where $X$ is some combination of constants (and further knowing that $g^{2} X$ is a renormalization group invariant [24, 29]), then

$$
P_{0} \equiv \sigma|\vec{y}|=\frac{g^{2} C_{F} X}{8 \pi}|\vec{y}|+\mathcal{O}(1 / m)
$$

The above result is that there exists a direct connection between the string tension and the nonperturbative YangMills sector of QCD at least under the truncation scheme considered here. The veracity of the truncation scheme will be discussed at the end.

\section{BETHE-SALPETER EQUATION: DIQUARKS}

Let us now briefly take a look at the diquark Bethe-Salpeter equation. The difference between this and the previously considered quark-antiquark system is actually rather simple from a technical standpoint, but leads to a completely different physical result. Recall that the quark and the antiquark propagators share the same Feynman prescription relative to their energy and only the constant components change. Thus, preserving the momentum routing of the Bethe-Salpeter equation, the result that the crossed box contributions to the Bethe-Salpeter kernel extends to the diquark case since this is purely dependent on the Feynman prescription. This means that we can immediately write down the Bethe-Salpeter equation for diquarks, at leading order in the mass expansion and within our truncation scheme:

$$
\begin{aligned}
\Gamma_{\alpha \beta}(p ; P)= & -\int d k \Gamma_{\bar{q} q \sigma \alpha \gamma}^{a}\left(p_{+},-k_{+}, k-p\right) W_{\sigma \sigma}^{a b}(p-k) \Gamma_{\bar{q} q \sigma \beta \delta}^{b}\left(-p_{-}, k_{-}, p-k\right) W_{\bar{q} q \gamma \lambda}\left(k_{+}\right) W_{\bar{q} q \delta \kappa}\left(-k_{-}\right) \Gamma_{\lambda \kappa}(k ; P) \\
& +\mathcal{O}(1 / m) .
\end{aligned}
$$

Again, the indices of the Bethe-Salpeter vertex function correspond to the quark content of the diquark and since the flavor and spin content decouple from the system, we shall only be interested in the color content of the diquark below. Expanding this out as before, we get the analogous result

$$
\left[P_{0}-2 m-g^{2} C_{F} \int_{r} \frac{\vec{\omega} \vec{\omega} D_{\sigma \sigma}(\vec{\omega})}{\vec{\omega}^{2}}\right] \Gamma_{\alpha \beta}(\vec{p} ; P)=g^{2} \int_{r} \frac{d \vec{\omega} D_{\sigma \sigma}(\vec{\omega})}{\vec{\omega}^{2}}\left[T^{a}\right]_{\alpha \lambda}\left[T^{a}\right]_{\beta \kappa} \Gamma_{\lambda \kappa}(\vec{p}-\vec{\omega} ; P)+\mathcal{O}(1 / m) .
$$


Fourier transforming as previously, and writing

$$
\left[T^{a}\right]_{\alpha \lambda}\left[T^{a}\right]_{\beta \kappa} \Gamma_{\lambda \kappa}(\vec{y})=C_{D} \Gamma_{\alpha \beta}(\vec{y})
$$

gives the solution

$$
P_{0}=2 m+g^{2} \int_{r} \frac{d \vec{\omega} D_{\sigma \sigma}(\vec{\omega})}{\vec{\omega}^{2}}\left\{C_{F}+e^{\imath \vec{\omega} \cdot \vec{y}} C_{D}\right\}+\mathcal{O}(1 / m) .
$$

The reappearance of the quark mass simply indicates that in contrast to the quark-antiquark system, there are now two co-moving quarks. The expression for the anti-diquark system is identical to the above, but with minus twice the mass - their velocities are simply reversed. The diquark is antisymmetric under interchange of the two quark legs and since the spin and flavor structure decouples, this means that the color structure must be antisymmetric. As for the quark-antiquark system, the system can only have a finite energy confining (energy increasing with separation) solution (if $C_{D}=-C_{F}$ ) or no finite solution at all. Using the Fierz identity Eq. (5.15), the color condition then reads

$$
-C_{F} \Gamma_{\alpha \beta}(\vec{y}) \equiv C_{D} \Gamma_{\alpha \beta}(\vec{y})=\frac{1}{2} \Gamma_{\beta \alpha}(\vec{y})-\frac{1}{2 N_{c}} \Gamma_{\alpha \beta}(\vec{y}) .
$$

Demanding the diquark color antisymmetry and with the definition Eq. (2.3) this becomes

$$
N_{c}^{2}-N_{c}-2=0 \quad \Rightarrow \quad N_{c}=-1,2 .
$$

So in $S U\left(N_{c}=2\right)$ there exists a confined, antisymmetric bound state of two quarks - the $S U(2)$ baryon and otherwise there are no (finite) physical states.

\section{SUMMARY, DISCUSSION AND CONCLUSIONS}

The connection between the Green's functions of Coulomb gauge Yang-Mills theory and physical quark confinement has been studied. Since all quarks are confined (irrespective of their mass), the generating functional of full QCD was expanded in the mass parameter and the leading order considered in a manner appropriate to Coulomb gauge and such that the system simplifies dramatically. This allowed the usage of the full nonperturbative quark equations of QCD in order to study the confinement properties. Using the Coulomb gauge Slavnov-Taylor identity for the quark-gluon vertices (whose derivation is one of the results of this study) and truncating the Yang-Mills sector to include only the nonperturbative gluon propagator, it was shown how the rainbow approximation to the quark and antiquark gap equations is exact in this case. It was then demonstrated that the corresponding ladder approximation to the Bethe-Salpeter equation was also exact. The mass expansion breaks the time-reversal and charge conjugation properties of the theory and in order to describe physical quark-antiquark states, the relevant Feynman prescription was introduced. With the analytic solutions to the gap equation, the Bethe-Salpeter equation was solved for the quark-antiquark and quark-quark channels. It was found that the only solutions correspond to confinement, namely that only color-singlet meson and $S U(2)$ baryon states have finite energy. This energy must increase linearly with separation either because: (i) there are no asymptotic quark states as is known in Coulomb gauge from the conserved and vanishing total charge [25], or (ii) as is suggested from the lattice results [32], the temporal propagator is infrared enhanced. Further, there exists a direct connection between the temporal gluon propagator of Yang-Mills theory and the string tension, at least within this truncation scheme.

Because of the (almost embarrassingly) simple results, it is worth re-emphasizing the input to the calculations. Different areas of study have been combined here: the heavy quark mass expansion, the Dyson-Schwinger formalism along with the Slavnov-Taylor identity and the Bethe-Salpeter equation, all of which have been considered within Coulomb gauge. Indeed, the simplification arising from this combination underscores how powerful the choice of Coulomb gauge is for nonperturbative studies. There are two approximations used. Firstly, the temporal gluon propagator is considered to be energy-independent. This is consistent with available lattice data [32], although explicitly in disagreement with perturbation theory [27]. Since this study is interested in the infrared properties of the theory, using the lattice suggestion seems justified. Actually, the connection between the explicit one-loop perturbative expressions and the leading order perturbative expansion (in covariant gauges, the resummation of the leading logarithms via the anomalous dimensions) is not yet understood in Coulomb gauge. Further, since the nonperturbative ghost propagator in Coulomb gauge is strictly independent of the energy [29] in order to cancel the closed ghost-loops occurring in the Yang-Mills expressions, the temporal gluon propagator must have some part that is also independent of the energy (the spatial gluon propagator is explicitly dependent on the energy). Thus it seems 
reasonable to assume that whatever energy dependence the temporal gluon propagator does have (and that is not visible with current lattice configurations), it is not important to the conclusions here.

The second approximation used in this study was to neglect the vertex and higher $n$-point functions of Yang-Mills theory. Recall that the tree-level spatial quark-gluon vertex is suppressed by the mass. This means that the number of truncated terms at leading order in the mass expansion is heavily suppressed. It was seen that without such terms, the quark-gluon vertices (and the Bethe-Salpeter kernel) reduced to their tree-level forms. This suggests that whilst the dressing function $D_{\sigma \sigma}$ does implicitly contain all nonperturbative effects associated with the dynamical dressing of the color charge (including, for example, potential glueball states), the quark-gluon vertices correspond to the naked quark color charge. One might express the results here as corresponding to a dressed color string confining two naked color sources. In the gap and Bethe-Salpeter equations, the explicit Yang-Mills contributions presumably cannot obliterate the effect of the rainbow-ladder exchange for a given temporal propagator input since (assuming the infrared enhancement as suggested from the lattice [32]) one would require an exact 'anti-confining' cancellation. Covariant gauge studies of the effect of non-Abelian corrections to the Bethe-Salpeter equation as applied to the light quark sector indicate that they are dominant compared to the Abelian corrections [11]. However, in the case studied here, there are no Abelian corrections and the diagrammatic content of the non-Abelian contribution is reduced by the mass expansion, making a quantitative comparison to known Bethe-Salpeter studies somewhat speculative. Perhaps a better comparison about the nature of neglecting the non-Abelian corrections is with Ref. [24], which refers to the Wilson loop of pure Yang-Mills theory. With effectively the same truncation (i.e., neglecting the Yang-Mills vertices), it was demonstrated that a string tension (the so-called Coulomb string tension) can also be extracted. Subsequently, it was shown that the physical string tension is lower than the Coulomb string tension [39] (a statement colloquially referred to as "no confinement without Coulomb confinement"). Thus, we anticipate that the effect of including the non-Abelian corrections to the formalism presented here would result not in the removal of the linearly rising bound state energy (i.e., not the cancellation of the ladder exchange) but rather in the softening of the coefficient by shifting the pole position by some finite amount. Physically, this amounts to the charge screening of the quark color charge.

The outlook of this study is extremely positive, with several avenues that might prove fruitful for further investigation. Maintaining the truncation scheme, one can consider the three quark (or two quark plus antiquark etc.) Faddeev equation to investigate $S U(3)$ baryons and whether or not the simple results for color confinement persist to the many-body system. Even more speculatively, given the simplicity of the leading order mass expansion one might imagine going even further and considering the two quark, two antiquark system. A second important line of study would be to consider the explicit inclusion of the Yang-Mills vertices to the combined system of Dyson-Schwinger, Slavnov-Taylor and Bethe-Salpeter equations, either phenomenologically or self-consistently to assess the influence of the truncation scheme considered so far. A third possibility is to investigate what happens when one chooses a different velocity parameter in the original decomposition of the quark fields. This would mix the temporal and spatial parts of the Coulomb gauge framework and allow one to investigate the connection between the respective gluon propagators.

\section{Acknowledgments}

The authors would like to thank G. Burgio for useful discussions. CP has been supported by the Deutscher Akademischer Austausch Dienst (DAAD). PW and HR have been supported by the Deutsche Forschungsgemeinschaft (DFG) under contracts no. DFG-Re856/6-2,3.

\section{Appendix A: Quark Slavnov-Taylor identity}

The derivation of the Slavnov-Taylor identity for the quark-gluon vertex will be presented in this Appendix. This derivation follows almost automatically as an extension to the Slavnov-Taylor identities for the Yang-Mills sector [29]. The full QCD action in the standard, second order formalism reads

$$
\mathcal{S}_{Q C D}=\int d^{4} x\left\{\bar{q}_{\alpha}\left[\imath \gamma^{0} \partial_{0}+g T^{a} \gamma^{0} \sigma^{a}+\imath \vec{\gamma} \cdot \vec{\nabla}-g T^{a} \vec{\gamma} \cdot \overrightarrow{A^{a}}-m\right]_{\alpha \beta} q_{\beta}-\frac{1}{4} F_{\mu \nu}^{a} F^{a \mu \nu}\right\}
$$

where the (antisymmetric) field strength tensor $F$ is given in terms of the gauge field $A_{\mu}^{a}$ :

$$
F_{\mu \nu}^{a}=\partial_{\mu} A_{\nu}^{a}-\partial_{\nu} A_{\mu}^{a}+g f^{a b c} A_{\mu}^{b} A_{\nu}^{c} .
$$

The action is invariant under a local $S U\left(N_{c}\right)$ gauge transform characterized by the parameter $\theta_{x}^{a}$ :

$$
U_{x}=\exp \left\{-\imath \theta_{x}^{a} T^{a}\right\}
$$


such that for infinitesimal $\theta_{x}^{a}$, the fields transform as (recall that $\sigma \equiv A_{0}$ )

$$
\begin{aligned}
& \sigma_{a} \rightarrow \sigma^{a}=\sigma^{a}-\frac{1}{g} \partial_{0} \theta^{a}-f^{a b c} \sigma^{b} \theta^{c}, \\
& \vec{A}^{a} \rightarrow \vec{A}^{\prime a}=\vec{A}^{a}+\frac{1}{g} \vec{\nabla} \theta^{a}-f^{a b c} \vec{A}^{b} \theta^{c}, \\
& q_{\alpha} \rightarrow q_{\alpha}^{\prime}=q_{\alpha}-\imath \theta^{a}\left[T^{a}\right]_{\alpha \beta} q_{\beta}, \\
& \bar{q}_{\alpha} \rightarrow \bar{q}_{\alpha}^{\prime}=\bar{q}_{\alpha}+\imath \theta^{a} \bar{q}_{\beta}\left[T^{a}\right]_{\beta \alpha} .
\end{aligned}
$$

Fixing to Coulomb gauge $\left(\vec{\nabla} \cdot \vec{A}^{a}=0\right)$ via the Faddeev-Popov technique introduces new terms into the action:

$$
\mathcal{S}_{F P}=\int d^{4} x\left[-\lambda^{a} \vec{\nabla} \cdot \vec{A}^{a}-\bar{c}^{a} \vec{\nabla} \cdot \vec{D}^{a b} c^{b}\right],
$$

where

$$
\vec{D}^{a b}=\delta^{a b} \vec{\nabla}-g f^{a c b} \vec{A}^{c}
$$

is the spatial covariant derivative (in the adjoint representation), $\lambda^{a}$ is a Lagrange multplier field to locally implement the gauge condition, $\bar{c}^{a}$ and $c^{b}$ are the Grassmann-valued ghost fields. The action is invariant under a Gauss-BRST transform [24] whereby the infinitesimal spacetime-dependent parameter $\theta_{x}^{a}$ is factorized into two Grassmann-valued components: $\theta_{x}^{a}=c_{x}^{a} \delta \lambda_{t}$, where $\delta \lambda_{t}$ is the time-dependent infinitesimal variation. The Gauss-BRST transform is a Coulomb gauge specific form of the standard BRS transform, allowed because the gauge-fixing term does not involve temporal differential operators. The variations of the new fields read:

$$
\delta \bar{c}^{a}=\frac{1}{g} \lambda^{a} \delta \lambda_{t}, \quad \delta c^{a}=-\frac{1}{2} f^{a b c} c^{b} c^{c} \delta \lambda_{t}, \quad \delta \lambda^{a}=0 .
$$

Including a source term,

$$
\mathcal{S}_{s}=\int d^{4} x\left[\rho^{a} \sigma^{a}+\vec{J}^{a} \cdot \vec{A}^{a}+\bar{c}^{a} \eta^{a}+\bar{\eta}^{a} c^{a}+\xi^{a} \lambda^{a}+\bar{q}_{\alpha} \chi_{\alpha}+\bar{\chi}_{\alpha} q_{\alpha}\right],
$$

the generating functional is given by

$$
Z[J]=\int \mathcal{D} \Phi \exp \left\{\imath \mathcal{S}_{Q C D}+\imath \mathcal{S}_{F P}+\imath \mathcal{S}_{s}\right\}
$$

The Coulomb gauge Slavnov-Taylor identities arise from regarding the Gauss-BRST transform as a change of integration variables under which the generating functional is invariant. Since the Jacobian factor is trivial [26] and only the source term varies, we deduce that

$$
\begin{aligned}
0= & \left.\int \mathcal{D} \Phi \frac{\delta}{\delta\left[\imath \delta \lambda_{t}\right]} \exp \left\{\imath \mathcal{S}_{Q C D}+\imath \mathcal{S}_{F P}+\imath \mathcal{S}_{s}+\imath \delta \mathcal{S}_{s}\right\}\right|_{\delta \lambda_{t}=0} \\
= & \int \mathcal{D} \Phi \exp \left\{\imath \mathcal{S}_{Q C D}+\imath \mathcal{S}_{F P}+\imath \mathcal{S}_{s}\right\} \int d^{4} x \delta\left(t-x_{0}\right)\left\{-\frac{1}{g}\left(\partial_{x}^{0} \rho_{x}^{a}\right) c_{x}^{a}+f^{a b c} \rho_{x}^{a} \sigma_{x}^{b} c_{x}^{c}\right. \\
& \left.-\frac{1}{g} J_{i x}^{a} \nabla_{i x} c_{x}^{a}+f^{a b c} J_{i x}^{a} A_{i x}^{b} c_{x}^{c}-\imath \bar{\chi}_{\alpha x} c_{x}^{a} T_{\alpha \beta}^{a} q_{\beta x}-\imath c_{x}^{a} \bar{q}_{\beta x} T_{\beta \alpha}^{a} \chi_{\alpha x}+\frac{1}{g} \lambda_{x}^{a} \eta_{x}^{a}+\frac{1}{2} f^{a b c} \bar{\eta}_{x}^{a} c_{x}^{b} c_{x}^{c}\right\} .
\end{aligned}
$$

Just as for the pure Yang-Mills case, the time dependence of the variation, $\delta \lambda_{t}$, results in the $\delta$-function constraint $\delta\left(t-x_{0}\right)$. The above identity is most usefully expressed in terms of proper functions and repeating the manipulations of Ref. [29], one arrives at the identity

$$
\begin{aligned}
0=\int d^{4} x \delta\left(t-x_{0}\right) & \left\{\frac{1}{g}\left(\partial_{x}^{0}<\imath \sigma_{x}^{a}>\right) c_{x}^{a}-f^{a b c}<\imath \sigma_{x}^{a}>\left[<\imath \rho_{x}^{b} \imath \bar{\eta}_{x}^{c}>+\sigma_{x}^{b} c_{x}^{c}\right]-\frac{1}{g}\left[\frac{\nabla_{i x}}{\left(-\nabla_{x}^{2}\right)}<\imath A_{i x}^{a}>\right]<\imath \bar{c}_{x}^{a}>\right. \\
& -f^{a b c}<\imath A_{i x}^{a}>t_{i j}(\vec{x})\left[<\imath J_{j x}^{b} \imath \bar{\eta}_{x}^{c}>+A_{j x}^{b} c_{x}^{c}\right]-\frac{1}{g} \lambda_{x}^{a}<\bar{c}_{x}^{a}>+\frac{1}{2} f^{a b c}<\imath c_{x}^{a}>\left[<\imath \bar{\eta}_{x}^{b} \imath \bar{\eta}_{x}^{c}>+c_{x}^{b} c_{x}^{c}\right] \\
& \left.+\imath T_{\alpha \beta}^{a}<\imath q_{\alpha x}>\left[<\imath \bar{\chi}_{\beta x} \imath \bar{\eta}_{x}^{a}>-c_{x}^{a} q_{\beta x}\right]+\imath T_{\beta \alpha}^{a}\left[<\imath \chi_{\beta x} \imath \bar{\eta}_{x}^{a}>+c_{x}^{a} \bar{q}_{\beta x}\right]<\imath \bar{q}_{\alpha x}>\right\} .
\end{aligned}
$$


Note that functional derivatives involving the Lagrange multiplier result merely in a trivial identity such that the classical field $\lambda_{x}^{a}$ can be set to zero [29]. Further, to derive the quark Slavnov-Taylor identities, one functional derivative with respect to $\imath c_{z}^{d}$ is needed and then the ghost fields/sources can be set to zero. Implementing this then gives

$$
\begin{aligned}
0=\int d^{4} x \delta\left(t-x_{0}\right) & \left\{-\frac{\imath}{g}\left(\partial_{x}^{0}<\imath \sigma_{x}^{d}>\right) \delta(z-x)-f^{a b c}<\imath \sigma_{x}^{a}>\left[\frac{\delta}{\delta \imath c_{z}^{d}}<\imath \rho_{x}^{b} \imath \bar{\eta}_{x}^{c}>-\imath \sigma_{x}^{b} \delta^{d c} \delta(z-x)\right]\right. \\
& +\frac{1}{g}\left[\frac{\nabla_{i x}}{\left(-\nabla_{x}^{2}\right)}<\imath A_{i x}^{a}>\right]<\imath \bar{c}_{x}^{a} \imath c_{z}^{d}>-f^{a b c}<\imath A_{i x}^{a}>t_{i j}(\vec{x})\left[\frac{\delta}{\delta \imath c_{z}^{d}}<\imath J_{j x}^{b} \imath \bar{\eta}_{x}^{c}>-\imath A_{j x}^{b} \delta^{d c} \delta(z-x)\right] \\
& -\imath T_{\alpha \beta}^{a}<\imath q_{\alpha x}>\left[\frac{\delta}{\delta \imath c_{z}^{d}}<\imath \bar{\chi}_{\beta x} \imath \bar{\eta}_{x}^{a}>+\delta^{d a} \delta(z-x) \imath q_{\beta x}\right] \\
& \left.+\imath T_{\beta \alpha}^{a}\left[\frac{\delta}{\delta \imath c_{z}^{d}}<\imath \chi_{\beta x} \imath \bar{\eta}_{x}^{a}>-\delta^{d a} \delta(z-x) \imath \bar{q}_{\beta x}\right]<\imath \bar{q}_{\alpha x}>\right\} .
\end{aligned}
$$

Two further functional derivatives with respect to $\imath q_{\gamma \omega}$ and $\imath \bar{q}_{\delta v}$ are taken and all remaing fields/sources set to zero. Given that [29]

$$
\frac{\delta}{\delta \imath c_{z}^{d}}<\imath \rho_{x}^{b} \imath \bar{\eta}_{x}^{c}>\left.\right|_{J=0}=t_{i j}(\vec{x}) \frac{\delta}{\delta \imath c_{z}^{d}}<\imath J_{j x}^{b} \imath \bar{\eta}_{x}^{c}>\left.\right|_{J=0}=0
$$

one obtains the Slavnov-Taylor identity for the quark-gluon vertices in configuration space:

$$
\begin{aligned}
0=\int d^{4} x \delta\left(t-x_{0}\right) & \left\{-\frac{\imath}{g}\left(\partial_{x}^{0}<\imath \bar{q}_{\delta v} \imath q_{\gamma \omega} \imath \sigma_{x}^{d}>\right) \delta(z-x)+\frac{1}{g}\left[\frac{\nabla_{i x}}{\left(-\nabla_{x}^{2}\right)}<\imath \bar{q}_{\delta v} \imath q_{\gamma \omega} \imath A_{i x}^{a}>\right]<\imath \bar{c}_{x}^{a} \imath c_{z}^{d}>\right. \\
& +\imath T_{\alpha \beta}^{a}<\imath \bar{q}_{\delta v} \imath q_{\alpha x}>\left[\frac{\delta^{2}}{\delta \imath q_{\gamma \omega} \delta \imath c_{z}^{d}}<\imath \bar{\chi}_{\beta x} \imath \bar{\eta}_{x}^{a}>\left.\right|_{J=0}+\delta^{d a} \delta(z-x) \delta_{\gamma \beta} \delta(\omega-x)\right] \\
& \left.+\imath T_{\beta \alpha}^{a}\left[\frac{\delta^{2}}{\delta \imath \bar{q}_{\delta v} \delta \imath c_{z}^{d}}<\imath \chi_{\beta x} \imath \bar{\eta}_{x}^{a}>\left.\right|_{J=0}-\delta^{d a} \delta(z-x) \delta_{\delta \beta} \delta(v-x)\right]<\imath \bar{q}_{\alpha x} \imath q_{\gamma \omega}>\right\}
\end{aligned}
$$

As with all Slavnov-Taylor identities, the above involves nontrivial ghost scattering-like kernels. Introducing some notation, these can be expressed via the Legendre transform in terms of a loop integral over proper Green's functions and propagators in the following way:

$$
\begin{aligned}
\tilde{\Gamma}_{\bar{q} ; \bar{c} c q \alpha \gamma}^{d}(x, z, \omega) \equiv & \imath g T_{\alpha \beta}^{a} \frac{\delta^{2}}{\delta \imath q_{\gamma \omega} \delta \imath c_{z}^{d}}<\imath \bar{\chi}_{\beta x} \imath \bar{\eta}_{x}^{a}>\left.\right|_{J=0} \\
= & \imath g T_{\alpha \beta}^{a}\left\{-<\imath \bar{\chi}_{\beta x} \imath \chi_{\varepsilon}><\imath \bar{q}_{\varepsilon} \imath q_{\gamma \omega} \imath \Phi_{\lambda}><J_{\lambda} \imath J_{\kappa}><\bar{\eta}_{x}^{a} \imath \eta_{\tau}><\imath \bar{c}_{\tau} \imath c_{z}^{d} \imath \Phi_{\kappa}>\right. \\
& \left.+<\imath \bar{\chi}_{\beta x} \imath \chi_{\kappa}><\imath \bar{q}_{\kappa} \imath q_{\gamma \omega} \imath \bar{c}_{\tau} \imath c_{z}^{d}><\imath \bar{\eta}_{x}^{a} \imath \eta_{\tau}>\right\} \\
\tilde{\Gamma}_{q ; \bar{c} c \bar{q} \delta \alpha}^{d}(x, z, v) \equiv & \frac{\delta^{2}}{\delta \imath \bar{q}_{\delta v} \delta \imath c_{z}^{d}}<\imath \chi_{\beta x} \imath \bar{\eta}_{x}^{a}>\left.\right|_{J=0} \imath g T_{\beta \alpha}^{a} \\
= & \left\{<\bar{\eta}_{x}^{a} \imath \eta_{\tau}><\imath \bar{c}_{\tau} \imath c_{z}^{d} \imath \Phi_{\kappa}><\imath J_{\kappa} \imath J_{\lambda}><\imath \bar{q}_{\delta v} \imath q_{\varepsilon} \imath \Phi_{\lambda}><\imath \bar{\chi}_{\varepsilon} \imath \chi_{\beta x}>\right. \\
& \left.-<\imath \bar{\eta}_{x}^{a} \imath \eta_{\tau}><\imath \bar{c}_{\tau} \imath c_{z}^{d} \imath \bar{q}_{\delta v} \imath q_{\kappa}><\imath \bar{\chi}_{\kappa} \imath \chi_{\beta x}>\right\} \imath g T_{\beta \alpha}^{a} .
\end{aligned}
$$

In the above, $J$ and $\Phi$ are dummy sources/fields refering to either $\vec{A}$ or $\sigma$ only, the internal indices otherwise refer to all attributes of the object in question (summed or integrated over). The configuration space Slavnov-Taylor identity can thus be written

$$
\begin{aligned}
0=\int d^{4} x \delta\left(t-x_{0}\right) & \left\{-\left(\imath \partial_{x}^{0}<\imath \bar{q}_{\delta v} \imath q_{\gamma \omega} \imath \sigma_{x}^{d}>\right) \delta(z-x)+\left[\frac{\nabla_{i x}}{\left(-\nabla_{x}^{2}\right)}<\imath \bar{q}_{\delta v} \imath q_{\gamma \omega} \imath A_{i x}^{a}>\right]<\imath \bar{c}_{x}^{a} \imath c_{z}^{d}>\right. \\
& +<\imath \bar{q}_{\delta v} \imath q_{\alpha x}>\left[\tilde{\Gamma}_{\bar{q} ; \bar{c} c q \alpha \gamma}^{d}(x, z, \omega)+\imath g T_{\alpha \gamma}^{d} \delta(z-x) \delta(\omega-x)\right] \\
& \left.+\left[\tilde{\Gamma}_{q ; \bar{c} c \bar{q} \delta \alpha}^{d}(x, z, v)-\imath g T_{\delta \alpha}^{d} \delta(z-x) \delta(v-x)\right]<\imath \bar{q}_{\alpha x} \imath q_{\gamma \omega}>\right\} .
\end{aligned}
$$

Defining the Fourier transform for the vertex functions (all momenta incoming):

$$
\Gamma(x, y, z)=\int d k_{1} d k_{2} d k_{3}(2 \pi)^{4} \delta\left(k_{1}+k_{2}+k_{3}\right) e^{-\imath k_{1} \cdot x-\imath k_{2} \cdot y-\imath k_{3} \cdot z} \Gamma\left(k_{1}, k_{2}, k_{3}\right),
$$


one can write the Slavnov-Taylor identity in momentum space (dropping the dirac and fundamental color indices for convenience)

$$
\begin{aligned}
& k_{3}^{0} \Gamma_{\bar{q} q \sigma}^{d}\left(k_{1}, k_{2}, k_{3}\right)=\imath \frac{k_{3 i}}{\vec{k}_{3}^{2}} \Gamma_{\bar{q} q A i}^{a}\left(k_{1}, k_{2}, k_{3}\right) \Gamma_{\bar{c} c}^{a d}\left(-k_{3}\right) \\
& \quad+\Gamma_{\bar{q} q}\left(k_{1}\right)\left[\tilde{\Gamma}_{\bar{q} ; \bar{c} c q}^{d}\left(k_{1}+q_{0}, k_{3}-q_{0}, k_{2}\right)+\imath g T^{d}\right]+\left[\tilde{\Gamma}_{q ; \bar{c} c \bar{q}}^{d}\left(k_{2}+q_{0}, k_{3}-q_{0}, k_{1}\right)-\imath g T^{d}\right] \Gamma_{\bar{q} q}\left(-k_{2}\right) .
\end{aligned}
$$

In the above, $k_{1}+k_{2}+k_{3}=0, \Gamma_{\bar{c} c}$ is the proper ghost two-point function and $q_{0}$ is the (arbitrary) energy injection scale that arises from the time-dependence of the Gauss-BRST transform. One can see the strong similarities of this expression with the Yang-Mills case [29]. In momentum space, the kernels can be written

$$
\begin{aligned}
\tilde{\Gamma}_{\bar{q} ; \bar{c} c q}^{d}\left(p_{1}, p_{2}, p_{3}\right)= & \iota g T^{a} \int d \omega W_{\bar{c} c}^{a b}(\omega) W_{\bar{q} q}\left(p_{1}-\omega\right) \\
& \times\left[\Gamma_{\bar{c} c \bar{q} q}^{b d}\left(\omega, p_{2}, p_{1}-\omega, p_{3}\right)-\Gamma_{\bar{c} c \kappa}^{b d c}\left(\omega, p_{2},-p_{2}-\omega\right) W_{\kappa \lambda}^{c e}\left(p_{2}+\omega\right) \Gamma_{\bar{q} q \lambda}^{e}\left(p_{1}-\omega, p_{3}, p_{2}+\omega\right)\right] \\
\tilde{\Gamma}_{q ; \bar{c} c \bar{q}}^{d}\left(p_{1}, p_{2}, p_{3}\right)= & \int d \omega\left[\Gamma_{\bar{c} c \kappa}^{b d c}\left(\omega, p_{2},-p_{2}-\omega\right) W_{\kappa \lambda}^{c e}\left(p_{2}+\omega\right) \Gamma_{\bar{q} q \lambda}^{e}\left(p_{3}, p_{1}-\omega, p_{2}+\omega\right)-\Gamma_{\bar{c} c \bar{q} q}^{b d}\left(\omega, p_{2}, p_{3}, p_{1}-\omega\right)\right] \\
& \times W_{\bar{c} c}^{a b}(\omega) W_{\bar{q} q}\left(\omega-p_{1}\right) \imath g T^{a}
\end{aligned}
$$

where the indices $\kappa, \lambda$, refer to the gluonic field types $\sigma$ or $\vec{A}$ (with the associated spatial index). Just as in the Yang-Mills case, the above Slavnov-Taylor identity, Eq. (A.18), in conjunction with the kernels can be solved in principle to give the temporal quark-gluon vertex $\Gamma_{\bar{q} q \sigma}$ in terms of purely spatial, ghost or quark propagators and proper functions. The identity is trivially satisfied at tree-level (using the Feynman rules of Ref. [28]) and can be verified at one-loop perturbatively (it is a purely technical exercise, so not suitable for presentation here).

[1] R. Sommer, Nucl. Phys. B 411, 839 (1994) [arXiv:hep-lat/9310022].

[2] M. Pak and H. Reinhardt, Phys. Rev. D 80, 125022 (2009) [arXiv:0910.2916 [hep-th]].

[3] M. Neubert, Phys. Rept. 245 (1994) 259 [arXiv:hep-ph/9306320].

[4] T. Mannel, Chin. J. Phys. 31 (1993) 1.

[5] B. Grinstein, "Lectures on heavy quark effective theory," Mexico City HE Phenom.1991:0161-217.

[6] E. Eichten and F. Feinberg, Phys. Rev. D 23, 2724 (1981).

[7] R. Alkofer, P. Watson and H. Weigel, Phys. Rev. D 65, 094026 (2002) [arXiv:hep-ph/0202053].

[8] P. Maris and P. C. Tandy, Phys. Rev. C 60, 055214 (1999) [arXiv:nucl-th/9905056].

[9] C. S. Fischer, J. Phys. G 32, R253 (2006) [arXiv:hep-ph/0605173].

[10] P. Watson, W. Cassing and P. C. Tandy, Few Body Syst. 35, 129 (2004) [arXiv:hep-ph/0406340].

[11] R. Williams, arXiv:0912.3494 [hep-ph].

[12] A. Bender, W. Detmold, C. D. Roberts and A. W. Thomas, Phys. Rev. C 65, 065203 (2002) [arXiv:nucl-th/0202082].

[13] M. S. Bhagwat, A. Holl, A. Krassnigg, C. D. Roberts and P. C. Tandy, Phys. Rev. C 70, 035205 (2004) [arXiv:nuclth/0403012].

[14] H. H. Matevosyan, A. W. Thomas and P. C. Tandy, Phys. Rev. C 75, 045201 (2007) [arXiv:nucl-th/0605057].

[15] A. Bender, C. D. Roberts and L. Von Smekal, Phys. Lett. B 380, 7 (1996) [arXiv:nucl-th/9602012].

[16] C. S. Fischer, P. Watson and W. Cassing, Phys. Rev. D 72, 094025 (2005) [arXiv:hep-ph/0509213].

[17] P. Watson and W. Cassing, Few Body Syst. 35, 99 (2004) [arXiv:hep-ph/0405287].

[18] C. S. Fischer and R. Williams, Phys. Rev. Lett. 103, 122001 (2009) [arXiv:0905.2291 [hep-ph]].

[19] S. L. Adler and A. C. Davis, Nucl. Phys. B 244, 469 (1984).

[20] R. Alkofer, M. Kloker, A. Krassnigg and R. F. Wagenbrunn, Phys. Rev. Lett. 96, 022001 (2006) [arXiv:hep-ph/0510028].

[21] E. S. Abers and B. W. Lee, Phys. Rept. 9, 1 (1973).

[22] V. N. Gribov, Nucl. Phys. B 139 (1978) 1.

[23] D. Zwanziger, Nucl. Phys. B 485, 185 (1997) [arXiv:hep-th/9603203].

[24] D. Zwanziger, Nucl. Phys. B 518 (1998) 237.

[25] H. Reinhardt and P. Watson, arXiv:0808.2436 [hep-th].

[26] P. Watson and H. Reinhardt, Phys. Rev. D 75, 045021 (2007) [arXiv:hep-th/0612114].

[27] P. Watson and H. Reinhardt, Phys. Rev. D 77, 025030 (2008) [arXiv:0709.3963 [hep-th]].

[28] C. Popovici, P. Watson and H. Reinhardt, Phys. Rev. D 79, 045006 (2009) [arXiv:0810.4887 [hep-th]].

[29] P. Watson and H. Reinhardt, Eur. Phys. J. C 65 (2010) 567 [arXiv:0812.1989 [hep-th]].

[30] P. Watson and H. Reinhardt, Phys. Rev. D 76, 125016 (2007) [arXiv:0709.0140 [hep-th]].

[31] G. Burgio, M. Quandt and H. Reinhardt, Phys. Rev. Lett. 102 (2009) 032002 [arXiv:0807.3291 [hep-lat]].

[32] M. Quandt, G. Burgio, S. Chimchinda and H. Reinhardt, PoS CONFINEMENT8, 066 (2008) [arXiv:0812.3842 [hep-th]]. 
[33] C. Feuchter and H. Reinhardt, Phys. Rev. D 70 (2004) 105021 [arXiv:hep-th/0408236]; C. Feuchter and H. Reinhardt, arXiv:hep-th/0402106.

[34] D. Epple, H. Reinhardt and W. Schleifenbaum, Phys. Rev. D 75, 045011 (2007) [arXiv:hep-th/0612241].

[35] D. Epple, H. Reinhardt, W. Schleifenbaum and A. P. Szczepaniak, Phys. Rev. D 77 (2008) 085007 [arXiv:0712.3694 [hep-th]].

[36] A. P. Szczepaniak and E. S. Swanson, Phys. Rev. D 65, 025012 (2002) [arXiv:hep-ph/0107078]; A. P. Szczepaniak, Phys. Rev. D 69, 074031 (2004) [arXiv:hep-ph/0306030].

[37] A. Cucchieri and D. Zwanziger, Phys. Rev. D 65, 014002 (2001) [arXiv:hep-th/0008248].

[38] J. S. Ball and T. W. Chiu, Phys. Rev. D 22, 2542 (1980).

[39] D. Zwanziger, Phys. Rev. Lett. 90, 102001 (2003) [arXiv:hep-lat/0209105]. 